\title{
Quasiperiodic functions and Dynamical Systems in Quantum Solid State Physics.
}

\author{
A.Ya.Maltsev ${ }^{1}$, S.P.Novikov ${ }^{1,2}{ }^{*}$ \\ (1) L.D.Landau Institute for Theoretical Physics, \\ 119334 ul. Kosygina 2, Moscow, \\ maltsev@itp.ac.ru , novikov@itp.ac.ru \\ (2) IPST, University of Maryland, \\ College Park MD 20742-2431,USA \\ novikov@ipst.umd.edu
}

\begin{abstract}
This is a survey article dedicated to the study of topological quantities in theory of normal metals discovered in the works of the authors during the last years. Our results are based on the theory of dynamical systems on Fermi surfaces. The physical foundations of this theory (the so-called "Geometric Strong Magnetic Field Limit") were found by the school of I.M.Lifshitz many years ago. Here the new aspects in the topology of quasiperiodic functions are developed.
\end{abstract}

\section{Introduction. Quasiperiodic functions and Dynamical Systems.}

According to the standard definition, quasiperiodic function of $l$ variables with $m$ quasiperiods is a restriction of any periodic function $f\left(y_{1}, \ldots, y_{m}\right)$ of $m$ variables on the $l$-dimensional affine subspace $R^{l} \subset R^{m}$.

Problem. What can one say about topology of the level curves of the quasiperiodic functions on the plane $R^{2}$ ?

*The work of S.P.Novikov is partially supported by the NSF Grant DMS 0072700. 
As we are going to demonstrate below, this problem has very important physical interpretation for the case where the number of quasiperiods is equal to $\mathrm{m}=3$. These studies motivated by the solid state physics, were started by S.P.Novikov in 1982 (see [12]). They were continued in his seminar (see $[16,13,14,19])$ as a nice purely topological investigations. In particular, an important breakthrough in the purely topological aspects of the "Novikov problem" for $m=3$ (i.e. exactly for the dimensions needed in physics) has been made by A.V.Zorich (see[16]) and I.A.Dynnikov ([19]). Long period no physical applications were expected. However, it was found later by the present authors (see $[20,26])$ that topological characteristics of this picture discovered in the previous works are organized in some sort of "topological resonance" leading to the important physical conclusions for the electric conductivity in the strong magnetic field. We shall explain these results below.

Let us explain a deep connection of the theory of quasiperiodic functions with special hamiltonian systems. Consider a torus $T^{m}$ as a factor-space of the Euclidean space $R^{m}$ with coordinates $p_{1}, \ldots, p_{m}$ by the lattice $\Gamma^{*}$ of the rank $m$. Let a constant Poisson Bracket be given on the torus by the skew-symmetric matrix $B^{i j}=-B^{j i}$ with rank equal to $2 s$. There are exactly $q=m-2 s$ linear "Casimirs" or "annihilator" functions $l_{1}, \ldots, l_{q}$ such that

$$
\left\{l_{a}, f(p)\right\}=B^{a b} \partial_{i} l_{a} \partial_{b} f=0
$$

Any hamiltonian function $\epsilon(\mathbf{p})$ on the torus $T^{m}$ determines a flow

$$
d p_{i} / d t=\left\{p_{i}, \epsilon\right\}=B^{a b} \partial_{a} p_{i} \partial_{b} \epsilon
$$

This system has only one well-defined one-valued integral of motion $\epsilon=$ const and $q$ multivalued conservative quantities $l_{j}=$ const, $j=1, \ldots, q$ generating a foliation on the torus. Let us point out that the restriction of the function $\epsilon(\mathbf{p})$ on the leaf of this foliation is exactly a quasiperiodic function with $m-q=2 s$ variables and $m$ quasiperiods.

We concentrated our studies on the special case $s=1$, i.e. rank of the Poisson Bracket is minimal possible (equal to 2). Here we have $2 s=2$, i.e. our quasiperiodic functions are defined on the 2-planes belonging to the family of parallel 2-planes in the space $R_{l}^{2} \subset R^{m}, l=l_{1} \ldots, l_{m-2}$ where $l_{j}=$ const, $j=1, \ldots, m-2$. We call energy levels by the "Fermi Surfaces" in this case. The restriction of the straight-line foliation on the surface $\epsilon=$ const gives trajectories of our hamiltonian system. They are obtained as sections 
of Fermi Surface by the planes $l_{j}=$ const, $j=1, \ldots, m-2$. We arrived to the following

Conclusion. Trajectories of the hamiltonian system described above exactly coincide with projections of the level curves of the quasiperiodic functions on the planes $R_{l}^{2} \subset R^{m}$ under the covering map $R^{m} \rightarrow T^{m}$.

As we shall see in the paragraph 2 , the case $m=3, q=1$ plays fundamental role in the solid state physics. The case $m=4, s=1$ also has been investigated. Some partial results were obtained in the work [30].

Definition 1 We call trajectory compact if it defines a compact curve on the plane $R^{2}$ as a level curve of the quasiperiodic function.

It follows from definition that periodic trajectories on Fermi surfaces in the 3-torus are compact if and only if they are homotopic to zero in the torus.

Definition 2 We call trajectory quasiperiodic with average direction $\eta$ if and only if corresponding level of quasiperiodic function $\epsilon=$ const on the plane $R_{l}^{2}$ lies in the strip of finite width between two straight lines parallel to the direction $\eta$. All trajectories more complicated than compact and quasiperiodic, we call chaotic.

In particular, all periodic trajectories non-homotopic to zero in the torus $T^{m}$, are quasiperiodic in that definition.

Definition 3 We call hamiltonian system "topologically completely integrable" on the given energy level if and only if all its trajectories are either compact or quasiperiodic on this level $\epsilon=$ const.

This definition has nothing common with the standard Liouville Complete Integrability.

Definition 4 We call quasiperiodic function topologically completely integrable if and only if all its levels are either compact or quasiperiodic. We call it stably completely integrable if it remains topologically completely integrable after any $C^{\infty}$-small perturbation of the m-periodic function $\epsilon\left(p_{1}, \ldots, p_{m}\right)$ and any small perturbation of the linear functions $l_{j}, j=1, \ldots, m-2$. 
This set of definitions gives only the first impression for the topological properties really needed for the physical applications. As it was found in the works $[20,26]$ where physical applications were obtained, there is a remarkable "topological resonance" of the topological quantities here extracted from the heart of the proofs of the Zorich and Dynnikov theorems, leading to the applications including the new observable topological phenomena:

Topological Resonance was found in [20] for the applications in physics. Quasiperiodic functions should be stably completely integrable for all family of parallel directions $l$. All quasiperiodic trajectories in this family should have the same average direction $\eta$. This direction should define an integral 2-plane $\eta \in Z^{2}$ in the reciprocal lattice $Z^{3}=\Gamma^{*}$. This integral 2-plane should be rigid under the small perturbations defining an open "Stability zone" on the sphere $S^{2}$ for the case $m=3, r=1$ where this plane is the same.

This topological resonance is valid for the generic directions, it is certainly untrue for the rational directions of magnetic field. The same definition works also for higher dimensions replacing 2-plane by the $(n-1)$-plane.

We shall return to the explicit formulation of corresponding results later. The exposition of topological theorems in the final form convenient for applications can be found with full set of proofs in [28] for the case $m=3, s=1$. Only partial results were obtained for the case $m=4, s=1$ (see[30]). As it was established in these cases, our hamiltonian system is generically stably completely integrable. Let $m=3, s=1$. More precisely, for the generic nonsingular Fermi surface $\epsilon=$ const the set of linear forms $l_{1} \in S^{2}$ ("directions of the magnetic fields" in physics) with chaotic dynamics has a Hausdorf dimension no more than 1 on the 2-sphere. In particular, its measure is equal to zero as it was proved by Dynnikov in 1999.

Conjecture. A Hausdorf dimension of the chaotic cases is strictly less than 1 for the physical case $m=1, s=1$.

For $m=4, s=1$ we presented an idea of the proof in [30] that the "stably integrable set" is open and dense in the Grassmanian $\left(l_{1}, l_{2}\right) \in G_{2,2}$ if the generic "Fermi level" $\epsilon=$ const is fixed in the space $T^{4}$. However, nothing is known about its measure. Our conjecture is that its measure is full, but no idea of the proof is known now.

We think that for $m \geq 5, s=1$ these systems are generically chaotic. 


\section{Normal Metals: The Standard Model.}

As it was well-known many years, even the ordinary electrical conductivity in single crystal normal metals cannot be explained properly without quantum mechanics. A working model for studying it has been elaborated in 1930s based on the quantum states of the free electron Fermi gas in some external 3D periodic potential created by the lattice $\Gamma$ of ions in the Euclidean space $R^{3}$. For the zero temperature $T=0$ our system lives in the standard Dirac-type ground state where all states with lowest energies are occupied (one electron for one state). The states of electron correspond exactly to the Bloch eigenfunctions of the one-particle Schrödinger operator $L \psi=\epsilon \psi$. By definition, we have

$$
\psi(\mathbf{x}+\mathbf{a})=\exp \{i<\mathbf{a}, \mathbf{p}>/ \hbar\} \psi(x), \quad \mathbf{a} \in \Gamma
$$

They depend on the "quasimomentum vector" $\mathbf{p}$ (or wave vector $\mathbf{k}=\mathbf{p} / \hbar$ ) belonging to the "Space of Quasimomenta"

$$
\mathbf{p} \in T^{3}=R^{3} / \Gamma^{*}
$$

where $\Gamma^{*}$ is a reciprocal lattice dual to the lattice $\Gamma$, i.e. for $\mathbf{p} \in \Gamma^{*}$ we have

$$
<\mathbf{a}, \mathbf{p}>=2 \pi \hbar n, \quad n \in Z
$$

For the given value of quasimomenta $p \in T^{3}$ there is a discrete spectrum of real energies $\epsilon_{n}(\mathbf{p}), n \geq 0$. They are called "Dispersion relations". According to the Fermi statistics, all levels $\epsilon<\epsilon_{F}$ are occupied for the zero temperature where the exact value $\epsilon_{F}$ (the "Fermi Energy") depends on the number of free electrons in the metal. We assume that this "Fermi level" $\epsilon(\mathbf{p})=\epsilon_{F}$ is a nondegenerate 2-manifold $S_{F}$ in the 3-torus of quasimomenta. It is called Fermi Surface. Physicists normally draw all pictures in the universal covering space $R^{3} \rightarrow T^{3}$ where we have a covering Fermi surface $\hat{S} \rightarrow S_{F}$ presented as a level of periodic function $\epsilon(\mathbf{p})$ of 3 variables $p_{1}, p_{2}, p_{3}$. We call it "a periodic surface". A fundamental ("Dirichle") domain of the lattice $\Gamma^{*}$ in $R^{3}$ people call "the first Brillouen zone" in the physics literature. For the temperature low enough the excited electron states are located in the small area nearby of Fermi level, so we continue to use a geometric picture 
described above. The limits for temperature should be discussed later. We are arriving to the conclusion that in the standard low-temperature model of normal metal the set of active electrons are identified with points of Fermi Surface $S_{F}$ in the Space of Quasimomenta $T^{3}$. This surface is orientable and homologous to zero in the 3-torus. Its topology can be complicated in some important cases like copper, gold, platinum and others. The most important topological characteristic of the connected piece of Fermi Surface is the Topological rank (introduced in [26]).

Definition 5 By the "Topological rank" $r$ of the connected piece of Fermi Surface we call a rank of the image-lattice $\pi_{1}\left(S_{F}\right) \rightarrow \pi_{1}\left(T^{3}\right)=Z^{3}$. Obviously we have $r=0,1,2,3$. We call connected Fermi surface topologically complicated if its topological rank is equal to 3.

Let us mention here that the first experimental observation of the Fermi surface corresponding to the Topological Rank 3 were made by Pippard for $C u([34])$.

Lemma 1 For any connected piece of Fermi Surface homologous to zero following inequality is true

$$
g \geq r
$$

where $r$ and $g$ are the topological rank and genus of Fermi surface correspondingly. For any connected piece of Fermi Surface non-homologous to zero the topological rank is no more than 2.

Proof. For $r=0,1$ the first case of this lemma is trivial. To prove it for $r=2,3$ we need to use that the homology class $i_{*}\left[S_{F}\right] \in H_{2}\left(T^{3}\right)$ is equal to zero for the embedding $i: S_{F} \rightarrow T^{3}$. Therefore the cohomological homomorphism $H^{2}\left(T^{3}\right) \rightarrow H^{2}\left(S_{F}\right)$ is also trivial. Therefore the product of any pair of classes

$$
y, z \in i^{*}\left(H^{1}\left(T^{3}\right)\right)=Z^{r} \subset H^{1}\left(S_{F}\right)=Z^{2 g}
$$

is equal to zero. The space $H^{1}\left(S_{F}\right)=Z^{2 g}$ is symplectic nondegenerate, and its subspace $i^{*}\left(H^{1}\left(T^{3}\right)=Z^{r}\right.$ is Lagrangian. Therefore we have

$$
2 g \geq 2 r
$$


Consider now any piece non-homologous to zero in the 3-torus. This submanifold $N_{F}^{0}$ in the 3 -torus is orientable. There exist 1-dimensional cycle in the 3-torus having nonzero intersection index with it. This cycle and its multiples do not belong to the image of the map $i_{*}: H_{1}\left(S_{F}^{0}\right) \rightarrow H_{1}\left(T^{3}\right)=Z^{3}$. Therefore its rank is no more than 2.

Our lemma is proved.

Let us remind here that for such noble metal as gold we have $r=3, g=4$.

\section{Electrons in the magnetic field. Dynamical Systems on Fermi Surfaces}

The classification of states described above works well in the absence of magnetic field. It is very difficult to study Schroedinger equation in the presence of magnetic field combined with periodic lattice potential. Nobody succeeded to find any suitable classification of the one-electron states if magnetic flux through elementary cell is irrational (in the natural quantum units) even for $2 \mathrm{D}$ crystals. For the real natural $3 \mathrm{D}$ crystals the size of elementary lattice is about $10^{-16} \mathrm{~cm}^{2}$. Therefore even the strong magnetic field of the order $1 t \sim 10^{4}$ Gauss gives only a small fraction of the quantum unit (about $10^{-3}$ ). Many years ago physicists developed a"semiclassical approach" to this problem where the dispersion relations and zone structure is taken exactly from the quantum theory and magnetic field is added "classically". The leading role in these studies since 1950s played Kharkov group of I.M.Lifshitz and his pupils (M.Azbel, M.Kaganov, V.Peschanski and others [1]-[3], see also [8]-[11]). It simply means that electrons start to move along the Fermi Surface in the space of quasimomenta $\mathbf{p} \in T^{3}=R^{3} / \Gamma^{*}$ with Poisson Bracket determined by the magnetic field

$$
\left\{p_{i}, p_{j}\right\}=\frac{e}{c} B^{i j}
$$

where $\epsilon_{i j k} B^{i j}=B_{k}$ is the ordinary vector of magnetic field dual to the skew symmetric tensor $B^{i j}$. This motion is generated by the hamiltonian $\epsilon(\mathbf{p})$ equal to the dispersion relation. We have

$$
d p_{i} / d t=\frac{e}{c} B^{i j} \partial_{j} \epsilon(\mathbf{p})=\frac{e}{c}[\nabla \epsilon(\mathbf{p}) \times \mathbf{B}]
$$


This system can be easily integrated analytically: in particular, its trajectories are exactly sections of the Fermi surface $S_{F}$ given by equation $\epsilon=\epsilon_{F}$, by the plane orthogonal to the vector $\mathbf{B}=\left(B_{1}, B_{2}, B_{3}\right)$ of magnetic field.

However, one should not think that this system is trivial because we have to identify points of the Euclidean space equivalent modulo reciprocal lattice. There are examples (constructed by S.Tsarev and I.Dynnikov-see in the survey article [23]) such that this system is chaotic.

Physicists of the Lifshitz group mentioned above formulated (and verified on the physical level) following fundamental

Geometric Strong Magnetic Field Limit: All essential properties of the electrical conductivity in the presence of the reasonably "strong" magnetic field (however, not exceeding the limits of semiclassical approximation) depend in main approximation on that dynamical system only.

The exposition of Kinetic Theory arguments leading to this conclusion can be found in the next paragraphs. In particular, these arguments lead to the values of magnetic field between one and several hundreds $t\left(1 t=10^{4}\right.$ Gauss $)$ for the real crystals like gold and temperatures like $T \sim 1 K$.

\section{Electron dynamics and Topological phenom- ena.}

Let us consider now in more details the physical phenomena connected with the geometry of quasi-classical electron orbits on the Fermi-surface. Namely, we are going to deal with the conductivity in normal metals in the presence of the strong homogeneous magnetic field $\mathbf{B}$. Let us explain first the concept of geometrical limit in this situation.

According to the standard approach we use the one-particle distribution function $f_{s}(\mathbf{p})$ defined on the three-dimensional torus $T^{3}$ for every energy

band $s$. The values of the functions $f_{s}(\mathbf{p})$ always belong to the interval $[0,1]$ for the fermions and the number of particles occupying the volume element $d^{3} p$ in the energy band $s$ can be written as

$$
d^{3} N_{s}=2 f_{s}(\mathbf{p}) \frac{d^{3} p}{(2 \pi \hbar)^{3}} V
$$

where $V$ is the total volume and the multiplier 2 is responsible for the spin 
degeneration. For the concentration of particles $n=N / V$ the analogous formula can then be written as

$$
d^{3} n_{s}=2 f_{s}(\mathbf{p}) \frac{d^{3} p}{(2 \pi \hbar)^{3}}
$$

In the absence of the external fields any distribution function $f_{s}(\mathbf{p})$ can be written in the form of well-known Fermi distribution corresponding to some fixed temperature $T$ :

$$
f_{s}^{T}(\mathbf{p})=\frac{1}{1+\exp \left(\frac{\epsilon_{s}(\mathbf{p})-\epsilon_{F}}{T}\right)}
$$

The parameter $\epsilon_{F}$ is called the Fermi energy of metal and can be defined from the total concentration of particles and the form of dispersion relations $\epsilon_{s}(\mathbf{p})$.

We are going to consider the situation of rather small temperatures $T \sim$ $1 K$ with respect to the width of the energy bands (usually $\epsilon_{\max }-\epsilon_{\min } \sim$ $\left.10^{4}-10^{5} \mathrm{~K}\right)$. We can put then $f_{s}(\mathbf{p}) \equiv 1$ for the energy bands lying completely below the Fermi energy $\epsilon_{F}$ and $f_{s}(\mathbf{p}) \equiv 0$ for the bands lying completely above $\epsilon_{F}$. Easy to see that this property will be conserved also in the presence of small external perturbations by energy reasons and only the bands with $\epsilon_{\min }<\epsilon_{F}<\epsilon_{\max }$ (conductivity bands) can be interesting for us. As can be easily seen from (2) all the functions $f_{s}(\mathbf{p})$ are smooth for $T>0$ and change rapidly from 0 to 1 in the narrow region $\sim T$ near the Fermi level $\epsilon_{s}(\mathbf{p})=\epsilon_{F}$ (see Fig. 1)

For $T=0$ we can put formally

$$
f_{s}(\mathbf{p})= \begin{cases}1 & \text { if } \quad \epsilon_{s}(\mathbf{p})<\epsilon_{F} \\ 0 & \text { if } \epsilon_{s}(\mathbf{p})>\epsilon_{F}\end{cases}
$$

though the zero temperature can not be obtained in the real experiments.

The total electric current for any given electron distribution can be calculated as the integral of group velocity $\mathbf{v}_{s}^{g r}(\mathbf{p})=\nabla \epsilon_{s}(\mathbf{p})$ over all the energy bands with the weights given by functions $f_{s}(\mathbf{p})$. We have so

$$
\mathbf{j}=2 \sum_{s} \int \ldots \int e \mathbf{v}_{T^{3}}^{g r}(\mathbf{p}) f_{s}(\mathbf{p}) \frac{d^{3} p}{(2 \pi \hbar)^{3}}=
$$




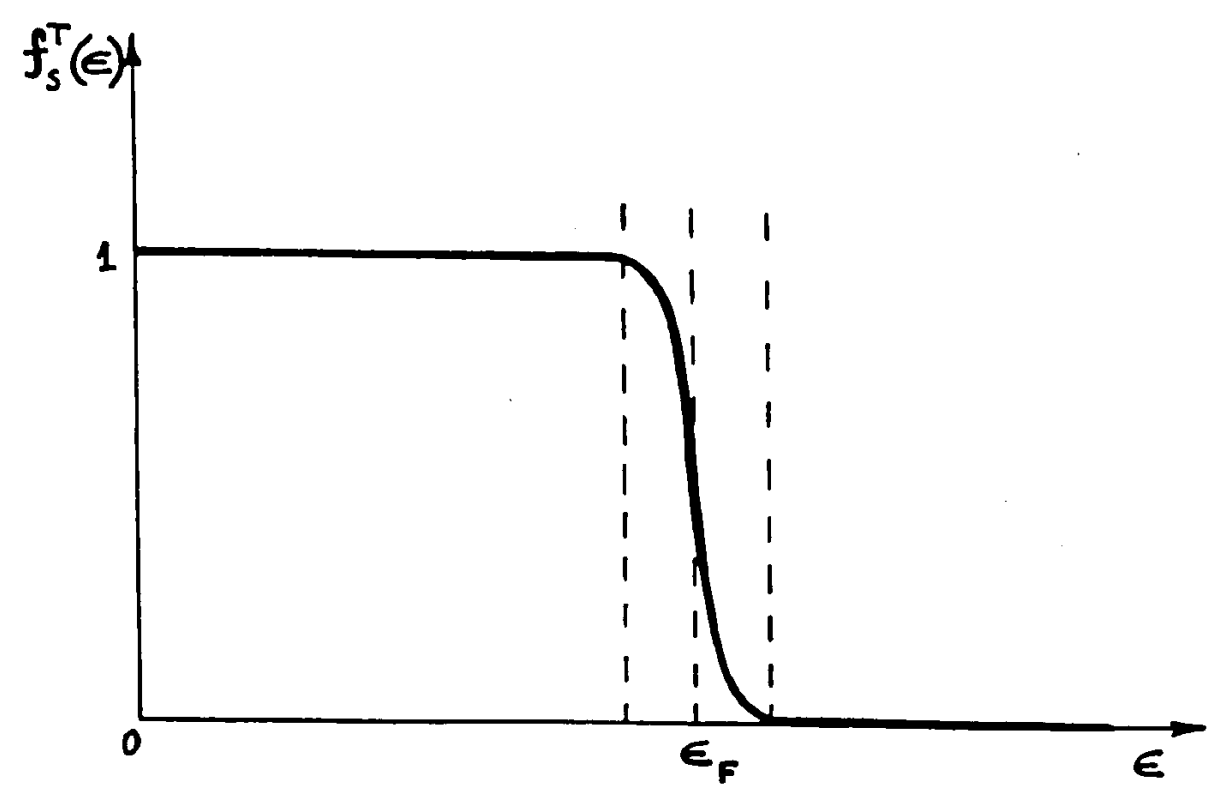

Figure 1: The Fermi distribution.

$$
=2 e \sum_{s} \int \ldots \int \nabla \epsilon_{T^{3}}(\mathbf{p}) f_{s}(\mathbf{p}) \frac{d^{3} p}{(2 \pi \hbar)^{3}}
$$

It is easy to see that all the functions $\mathbf{v}_{s}^{g r}(\mathbf{p})$ are the odd functions on the tori $T^{3}\left(\epsilon_{s}(\mathbf{p})=\epsilon_{s}(-\mathbf{p})\right)$ and the total electric current is zero for any Fermi distribution $f_{s}^{T}(\mathbf{p})$ given by $(2)$.

In our quasiclassical approach we will neglect the quantization of the electron energy levels in the magnetic field $\mathbf{B}$ and use just the classical system (1) to describe the electron behavior in the presence of magnetic field. Let us just point out here that for $\hbar \omega_{B} \ll \epsilon_{F}$ the quantization will not change the geometric characteristics of conductivity for rather strong magnetic fields.

Let us note now that the dynamical system (1) does not change any distribution (2) since both the energy $\epsilon_{s}(\mathbf{p})$ and the volume element $d^{3} p$ are conserved by this system. However, the form of the linear response to the small electric field $\mathbf{E}$ depends strongly on the geometry of the trajectories of (1) as we will see below.

We define the Fermi surface $S_{F}$ as the union of all surfaces given by the equation $\epsilon_{s}(\mathbf{p})=\epsilon_{F}$ for all conductivity bands. It can be shown that these parts do not intersect each other in the generic situation by quantum mechanical reasons and the Fermi surface can be usually represented as a disjoint union of smooth compact nonselfintersecting pieces in the three-dimensional 
torus $T^{3}$. The Fermi surface $S_{F}$ is homologous to zero in $T^{3}$ by construction and all the components of $S_{F}$ give the independent contribution to the conductivity tensor $\sigma^{i k}$. We can consider then separately every conductivity zone with the dispersion relation $\epsilon(\mathbf{p})=\epsilon_{s}(\mathbf{p})$. The corresponding contributions to the conductivity should then just be added in the three dimensional tensor $\sigma^{i k}$. The forms of these contributions will however use also the fact of nonselfintersecting of different components of the Fermi surface as will follow from the Topological consideration. We will discuss later also the case when for some special reason different components of $S_{F}$ can intersect each other.

Let us describe now the "Geometric Strong Magnetic Field limit" of conductivity in our situation.

We will introduce the mean electron free motion time $\tau$ characterizing the mean time interval of the free electron motion between the two scattering acts. The time $\tau$ is defined just by scattering on the impurities for rather low temperatures $T$ and depends on the purity of the crystal. We can assume then that every electron lives on the same trajectory of (1) during the mean time $\tau$ and change the trajectory after the scattering act. The geometric length of the corresponding trajectory interval $l_{p}$ (in $\mathbf{p}$-space) is proportional to the magnetic field $B$ and tends to the infinity as $B \tau \rightarrow \infty$. We can see then that any small perturbation of the Fermi distribution (2) will be instantly "mixed" along the trajectories of (1) in this strong magnetic field limit. All the averaged values should thus then be calculated in this situation for the "averaged" distribution constant on the trajectories of (2). All stationary distributions should also be constant on the trajectories of (1) as $B \tau \rightarrow \infty$ and can just be slightly different from these constants for large finite values of $B \tau$. As was shown in [1] these distributions can be expanded as regular functions in powers of $(B \tau)^{-1}$ in this limit for closed and open periodic electron orbits. This approach will work well also for more general cases of regular stable open orbits giving the similar effects in this case. However, as we will see below, it can not be applied in more complicated "chaotic" behavior of the electron orbits and the situation is much more complicated in this case.

We will call the Geometric Strong Magnetic Field Limit the situation when $l_{p} \gg p_{0}$ where $p_{0}$ is the size of the Brillouen zone in the $\mathbf{p}$-space. For the crystal lattice with the lattice constant $\sim a$ we will have $p_{0} \sim 2 \pi \hbar / a$. Let us introduce the cyclotron frequency $\omega_{B}=e B / m^{*} c$ where $m^{*}$ is the "effective mass" of electron in the crystal. Using the standard approximation 

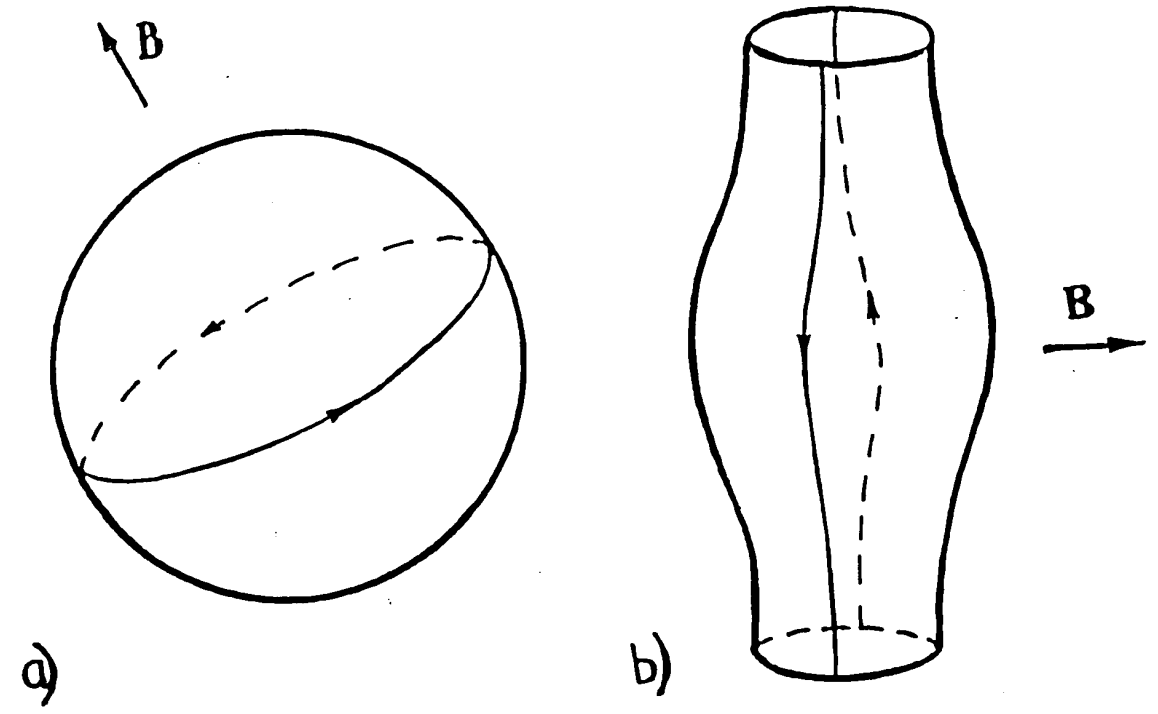

Figure 2: The simplest closed and open periodic trajectories.

$m^{*} v_{g r} \sim p_{F} \sim p_{0}$ on the Fermi surface we can write the condition of strong magnetic field limit in usual form $\omega_{B} \tau \gg 1$.

The electron dynamics in $\mathbf{x}$-space can be described by additional system

$$
\dot{\mathbf{x}}=\mathbf{v}_{g r}(\mathbf{p})
$$

(in quasiclassical approach) and can be easily reconstructed for any known trajectory of (1) in $\mathbf{p}$-space. Let us choose now the $z$-axis along the direction of the magnetic field $\mathbf{B}$ while the $x y$-plane will be orthogonal to $\mathbf{B}$. It is easy to see then that the $x y$ projection of the electron trajectory in $\mathrm{x}$-space can be obtained just by rotation of corresponding trajectory of (1) by $\pi / 2$. As a corollary of this fact the asymptotic behavior of conductivity in the plane orthogonal to $\mathbf{B}$ is defined completely by the geometry of the electron orbits in $\mathbf{p}$-space $([1]-[3])$.

Let us consider here the closed and the open periodic electron trajectories in the $\mathbf{p}$-space $([1])$. As easy to see the closed trajectories can arise in many different situations. The open periodic trajectories can be obtained for instance as the intersection of the periodic cylinder in $\mathbf{p}$-space by the plane containing the vector parallel to the axis of cylinder (see Fig 2. a,b).

We note here that the open periodic trajectories always come in pairs with opposite parallel directions as follows from the fact that the Fermi surface is homologous to zero in $T^{3}$. 
Let us choose the $x$-axis along the mean direction of the open orbits in $\mathbf{p}$-space in the plane orthogonal to $\mathbf{B}$ for the second situation and arbitrarily in the plane orthogonal to $\mathbf{B}$ for the case of closed electron orbits only.

The projection of the mean direction of open orbits in the plane orthogonal to $\mathbf{B}$ in $\mathbf{x}$-space will be directed along the $y$-axis according to our remark above.

The corresponding asymptotic behavior of 3-dimensional conductivity tensor can then be written in the following form ( [1]):

Case 1 (closed orbits):

$$
\sigma^{i k} \simeq \frac{n e^{2} \tau}{m^{*}}\left(\begin{array}{ccc}
\left(\omega_{B} \tau\right)^{-2} & \left(\omega_{B} \tau\right)^{-1} & \left(\omega_{B} \tau\right)^{-1} \\
\left(\omega_{B} \tau\right)^{-1} & \left(\omega_{B} \tau\right)^{-2} & \left(\omega_{B} \tau\right)^{-1} \\
\left(\omega_{B} \tau\right)^{-1} & \left(\omega_{B} \tau\right)^{-1} & *
\end{array}\right)
$$

Case 2 (open periodic orbits):

$$
\sigma^{i k} \simeq \frac{n e^{2} \tau}{m^{*}}\left(\begin{array}{ccc}
\left(\omega_{B} \tau\right)^{-2} & \left(\omega_{B} \tau\right)^{-1} & \left(\omega_{B} \tau\right)^{-1} \\
\left(\omega_{B} \tau\right)^{-1} & * & * \\
\left(\omega_{B} \tau\right)^{-1} & * & *
\end{array}\right)
$$

Here $\simeq$ means " of the same order in $\omega_{B} \tau$ and $*$ are some constants $\sim 1$. Let us mention also that the relations (3)-(4) give only the absolute values of $\sigma^{i k}$.

More complicated types of open electron orbits were constructed in [2][3]. Let represent here these results in a brief form.

"Thin spatial net".

The form of the thin spatial net is shown on the Fig. 3,a. The net corresponds to the cubic symmetry of the crystal and the thickness of tubes is considerably smaller than the periods of the net.

We can parameterize now the directions of $\mathbf{B}$ by the points of the unit sphere $S^{2}$ and try to find those directions for which we have the open electron orbits on the net. As was pointed out in [2] the open electron orbits exist in this case only for six small regions on the unit sphere close to the main crystallographic directions $( \pm 1,0,0),(0, \pm 1,0)$ and $(0,0, \pm 1)$ (see Fig. 3b). For the directions of $\mathbf{B}$ lying out of these domains the open electron orbits do not appear. Let us mention that this type of trajectories is obviously different from that shown on Fig. 2b. This circumstance can be easily seen from the 

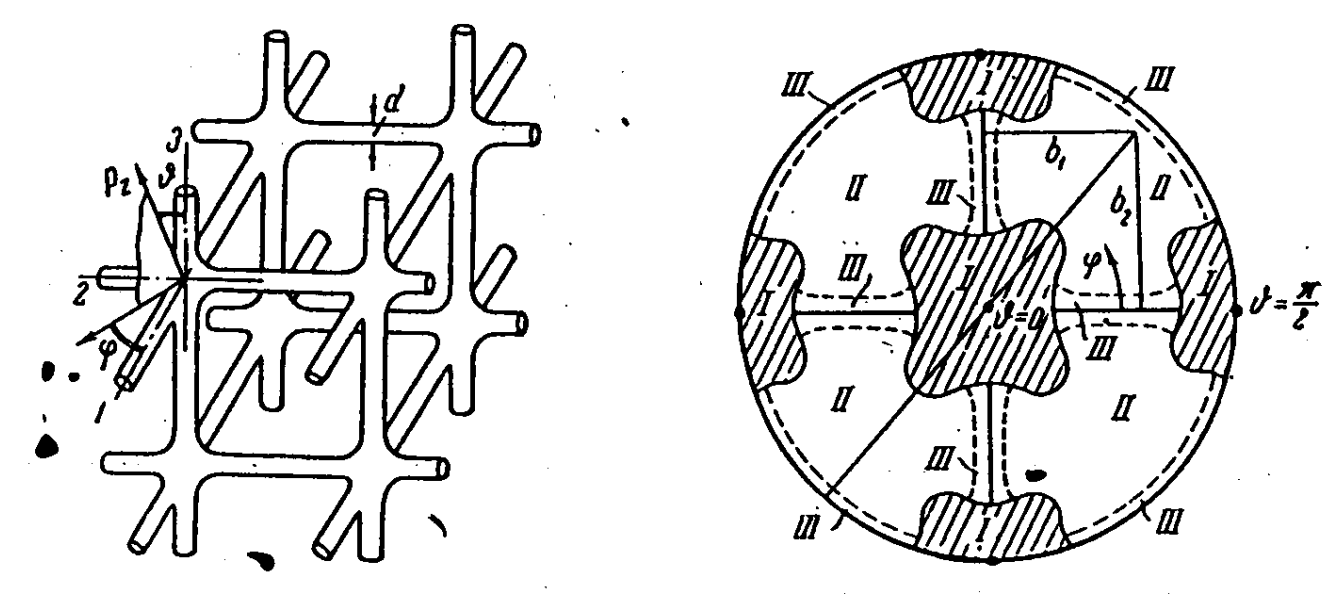

Figure 3: The "thin spatial net" and the corresponding zones on the unit sphere where the open trajectories exist. As was observed in [2] the mean directions of open orbits are given by the intersections of planes orthogonal to $\mathbf{B}$ with the coordinate planes $x y, y z, x z$.

fact that in the case of periodic ("warped") cylinder the open trajectories exist only for the directions of $\mathbf{B}$ orthogonal to the axis of cylinder and do not appear for any other direction. In the case of thin spatial net we now have the whole regions on the unit sphere corresponding to non-closed orbits. These new trajectories are not periodic anymore. However, it was shown in [2] that they all have the mean asymptotic directions given by the intersections of the corresponding planes $\Pi(\mathbf{B})$ orthogonal to $\mathbf{B}$ with the coordinate planes $x y, y z$ and $x z$ (orthogonal to the corresponding main crystallographic directions). Let us pay here the special attention to the last two circumstances. We will come back to these facts when discuss the general topological approach to the classification problem.

As was stated in [2] the corresponding contribution to the conductivity can be also written for such orbits in the form (4) with the $x$-axis directed along the asymptotic direction of trajectories in $\mathbf{p}$-space.

Now let us represent also the results of [3] concerning the analytical Fermi surfaces given by the finite-parametric family of the form:

$$
\begin{gathered}
\alpha\left(\cos \frac{a p_{x}}{\hbar}+\cos \frac{a p_{y}}{\hbar}+\cos \frac{a p_{z}}{\hbar}\right)+ \\
+\beta\left(\cos \frac{a p_{x}}{\hbar} \cos \frac{a p_{y}}{\hbar}+\cos \frac{a p_{y}}{\hbar} \cos \frac{a p_{z}}{\hbar}+\cos \frac{a p_{x}}{\hbar} \cos \frac{a p_{z}}{\hbar}\right)+
\end{gathered}
$$




$$
+\delta \cos \frac{a p_{x}}{\hbar} \cos \frac{a p_{y}}{\hbar} \cos \frac{a p_{z}}{\hbar}=\zeta_{0}
$$

The form of the Fermi surface will now depend on the values of the parameters $\alpha, \beta, \delta$ and $\zeta_{0}$. As was shown in [3] the open electron orbits exist in this case for four additional different topological types of the Fermi surfaces given by (5) (excluding the spatial net described above). According to [3] the open orbits exist in these cases in open zones and on the onedimensional curves on the unit sphere represented on the Fig. 4.

We will discuss later the general topological classification of arbitrary complicated Fermi surfaces. Let us just make some comments about the picture on Fig. 4. The dark domains on the Fig. 4 give the positions of the largest zones corresponding to open orbits for the given topological types of Fermi surface. These domains always exist for the Fermi surfaces described above being connected with the symmetry of dispersion relation and the topology of the Fermi surface. However, as we will discuss later, without any requirements on the "thickness of tubes" the topological form of the Fermi surface itself does not determine all the regions on the unit sphere corresponding to the open electron orbits. So, in all the cases on Fig. 4 we predict also an infinite number of smaller zones on the unit sphere corresponding to open orbits which forms and positions will depend on the parameters $\alpha, \beta, \delta$ and $\zeta_{0}$. Besides that, there will be some special "unstable" points on the unit sphere where the very complicated open orbits can exist being completely unstable w.r.t. the small rotations of the magnetic field and change of parameters $\alpha, \beta, \delta, \zeta_{0}$.

Let us also point out here the mistake on the last picture of Fig. 4 connected with the overlapping of the domains corresponding to open orbits. It was claimed in [3] that the open regions with different mean directions exist in the intersections of dark domains on the last picture. However, this situation contradicts to the Topological Resonance phenomenon which we will discuss below. As we will see then these domains can not intersect each other over the whole open regions on the unit sphere. So we claim that the dark regions on the last picture should be actually smaller and do not overlap each other.

Let us consider now the general topological approach to the classification of the open orbits for arbitrary smooth three-periodic Fermi surfaces in $R^{3}$.

The general problem of classification of quasiclassical electron orbits for 

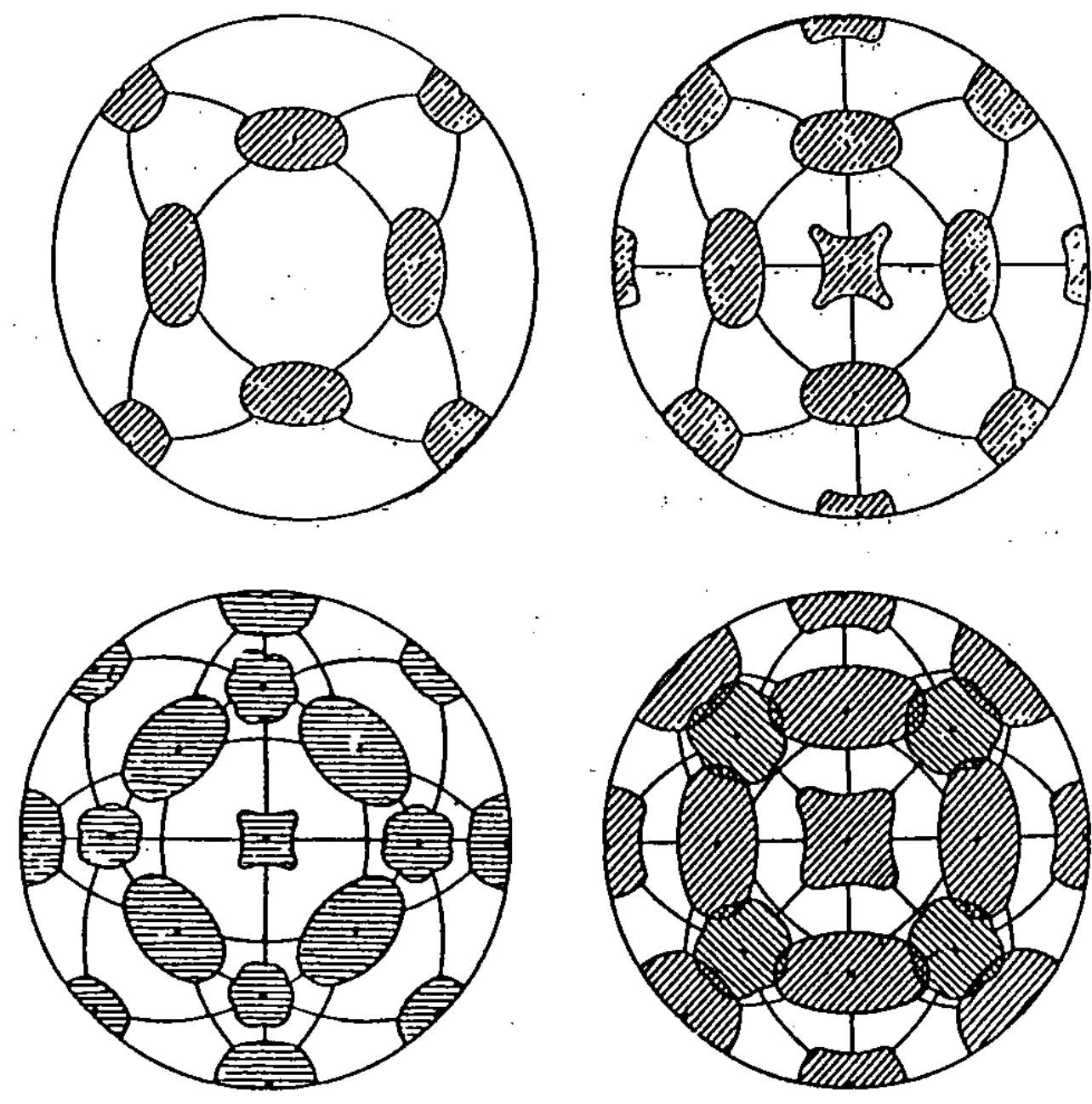

Figure 4: The open zones and the one-dimensional circles on the unit sphere corresponding to open orbits for four different Fermi surfaces from the family (5) represented in [3]. The last picture contains a conceptual mistake contradicting to Topological Resonance phenomenon. Namely, the angle diagram can not contain the whole open domains on the unit sphere where the open trajectories with different mean direction exist. 
arbitrary Fermi surface was set by S.P.Novikov in [12]. This problem has been studied by his pupils since 1980's. The important contribution was made by A.V.Zorich, I.A.Dynnikov and S.P.Tsarev. During this period the deep topological results were obtained which form now the modern understanding of situation. The general picture is rather non-trivial and includes the generic behavior and the special degenerate cases. During the last years the valuable numerical calculations were done also by R.D.Leo ([31]).

In particular, using the topological resonance following from the proofs of these theorems the present authors invented the so-called "Topological quantum numbers" observable in the conductivity of normal metals ([20]). These characteristics are represented by the integral planes connected with zones on the unit sphere corresponding to open orbits. The total set of such planes together with the geometry of corresponding zones gives the important topological characteristic of the dispersion relation in metal.

It was shown also (S.P.Tsarev, I.A.Dynnikov) that the chaotic open orbits can also exist and reveal much more complicated behavior. We will discuss later these cases in details.

Before going further let us introduce the basic definitions for the complicated Fermi surfaces in $R^{3}$.

Definition 6 I) Genus.

Let us now come back to the original phase space $T^{3}=R^{3} / \Gamma^{*}$. The reciprocal lattice $\Gamma^{*}$ is generated by the vectors $\mathbf{g}_{1}, \mathbf{g}_{2}, \mathbf{g}_{3}$ connected with the vectors $\mathbf{l}_{1}, \mathbf{l}_{2}, \mathbf{l}_{3}$ of the physical lattice $\Gamma$ by the simple formulas:

$$
\mathbf{g}_{1}=2 \pi \hbar \frac{\mathbf{l}_{2} \times \mathbf{l}_{3}}{\left(\mathbf{l}_{1}, \mathbf{l}_{2}, \mathbf{l}_{3}\right)}, \mathbf{g}_{2}=2 \pi \hbar \frac{\mathbf{l}_{3} \times \mathbf{l}_{1}}{\left(\mathbf{l}_{1}, \mathbf{l}_{2}, \mathbf{l}_{3}\right)}, \quad \mathbf{g}_{3}=2 \pi \hbar \frac{\mathbf{l}_{1} \times \mathbf{l}_{2}}{\left(\mathbf{l}_{1}, \mathbf{l}_{2}, \mathbf{l}_{3}\right)}
$$

Every component of the Fermi surface becomes then the smooth orientable 2-dimensional surface embedded in $T^{3}$. We can then introduce the standard genus of every component of the Fermi surface $g=0,1,2, \ldots$ according to standard topological classification depending on if this component is topological sphere, torus, sphere with two holes, etc ... .

II) Topological Rank. 


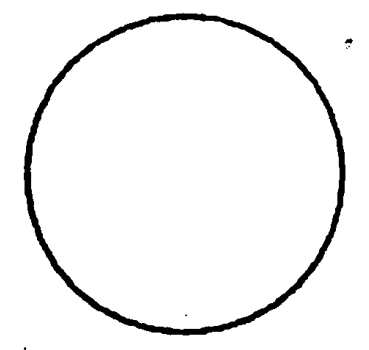

(a)
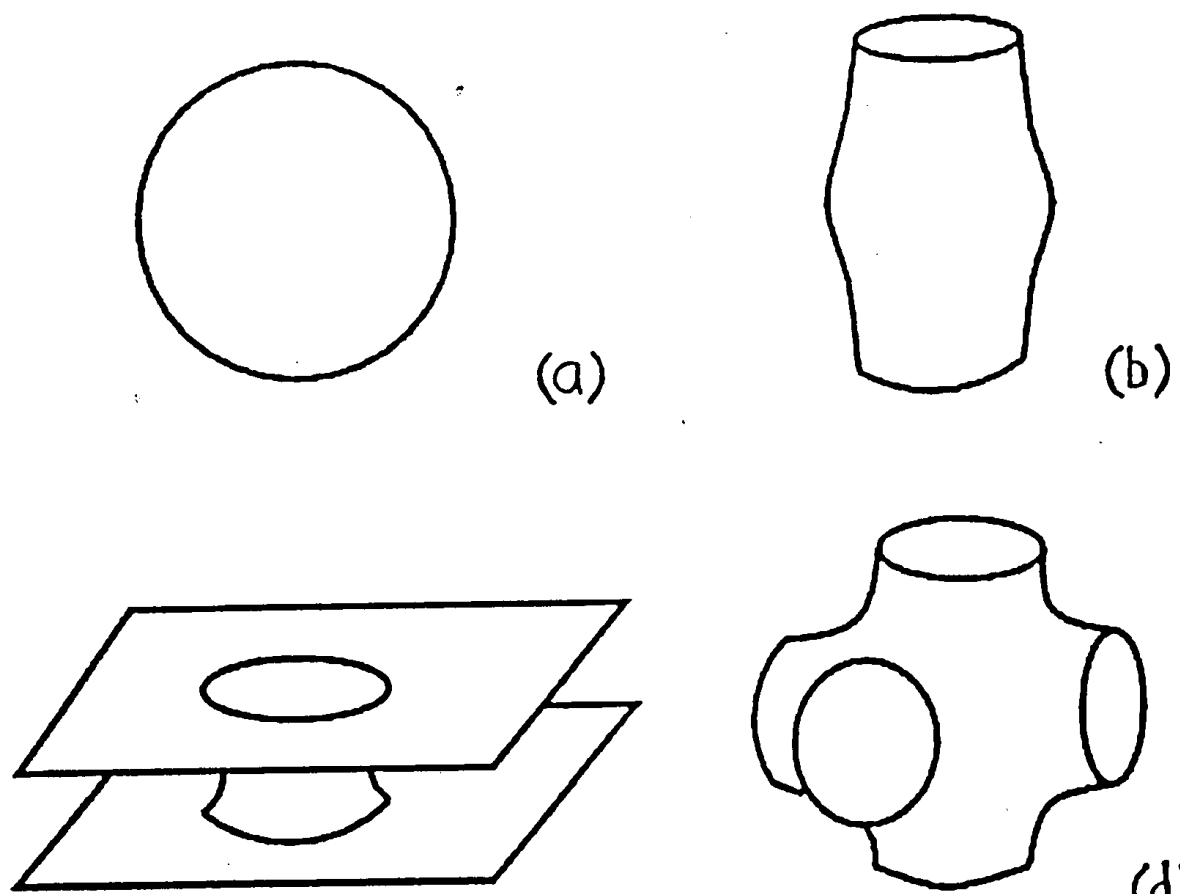

(c)

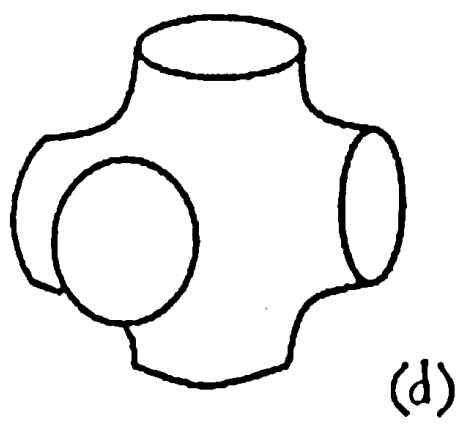

Figure 5: The Fermi surfaces with Topological Ranks 0, 1, 2 and 3 respectively.

Let us introduce the Topological Rank $r$ as the characteristic of the embedding of the Fermi surface in $T^{3}$. It's much more convenient in this case to come back to the total $\mathbf{p}$-space and consider the connected components of the three-periodic surface in $R^{3}$.

1) The Fermi surface has Rank 0 if every its connected component can be bounded by a sphere of finite radius.

2) The Fermi surface has Rank 1 if every its connected component can be bounded by the periodic cylinder of finite radius and there are components which can not be bounded by the sphere.

3) The Fermi surface has Rank 2 if every its connected component can be bounded by two parallel (integral) planes in $R^{3}$ and there are components which can not be bounded by cylinder.

4) The Fermi surface has Rank 3 if it contains components which can not be bounded by two parallel planes in $R^{3}$.

The pictures on Fig. 5, a-d represent the pieces of the Fermi surfaces in $R^{3}$ with the Topological Ranks $0,1,2$ and 3 respectively.

It is easy to see also that the topological Rank coincides with the max- 
imal Rank of the image of mapping $\pi_{1}\left(S^{i}\right) \rightarrow \pi_{1}\left(T^{3}\right)$ for all the connected components of the Fermi surface.

As can be seen the genuses of the surfaces represented on the Fig. 5, a-d are also equal to 0, 1, 2 and 3 respectively. However, the genus and the Topological Rank are not necessary equal to each other in the general situation.

Let us discuss briefly the connection between the genus and the Topological Rank since this will play the crucial role in further consideration. It is easy to see that the Topological Rank of the sphere can be only zero and the Fermi surface consists in this case of the infinite set of the periodically repeated spheres $S^{2}$ in $R^{3}$.

The Topological Rank of the torus $T^{2}$ can take three values $r=0, r=1$ and $r=2$.

It is easy to see that all the three cases of periodically repeated tori $T^{2}$ in $R^{3}$, periodically repeated "warped" integral cylinders and the periodically repeated "warped" integral planes give the topological 2-dimensional tori $T^{2}$ in $T^{3}$ after the factorization. (Let us note here that we call the cylinder in $R^{3}$ integral if it's axis is parallel to some vector of the reciprocal lattice, while the plane in $R^{3}$ is called integral if it is generated by some two reciprocal lattice vectors.) The case $r=2$, however, has an important difference from the cases $r=0$ and $r=1$. The matter is that the plane in $R^{3}$ is not homological to zero in $T^{3}$ (i.e. does not restrict any domain of "lower energies") after the factorization. We can conclude so that if these plains appear as the connected components of the physical Fermi surface they should always come in pairs, $\Pi_{+}$and $\Pi_{-}$, which are parallel to each other in $R^{3}$. The factorization of $\Pi_{+}$ and $\Pi_{-}$gives then the two tori $T_{+}^{2}, T_{-}^{2}$ with the opposite homological classes in $T^{3}$ after the factorization. The space between the $\Pi_{+}$and $\Pi_{-}$in $R^{3}$ can now be taken as the domain of lower (or higher) energies and the disjoint union $\Pi_{+} \cup \Pi_{-}$will correspond to the union $T_{+}^{2} \cup T_{-}^{2}$ homological to zero in $T^{3}$.

It can be shown that the Topological Rank of any component of genus 2 can not exceed 2 also. The example of the corresponding immersion of such component with maximal Rank is shown at Fig. 5, c and represents the two parallel planes connected by cylinders.

At last we say that the Topological Rank of the components with genus $g \geq 3$ can take any value $r=0,1,2,3$.

Let us also show at last two "exotic examples" of the Fermi surfaces of 

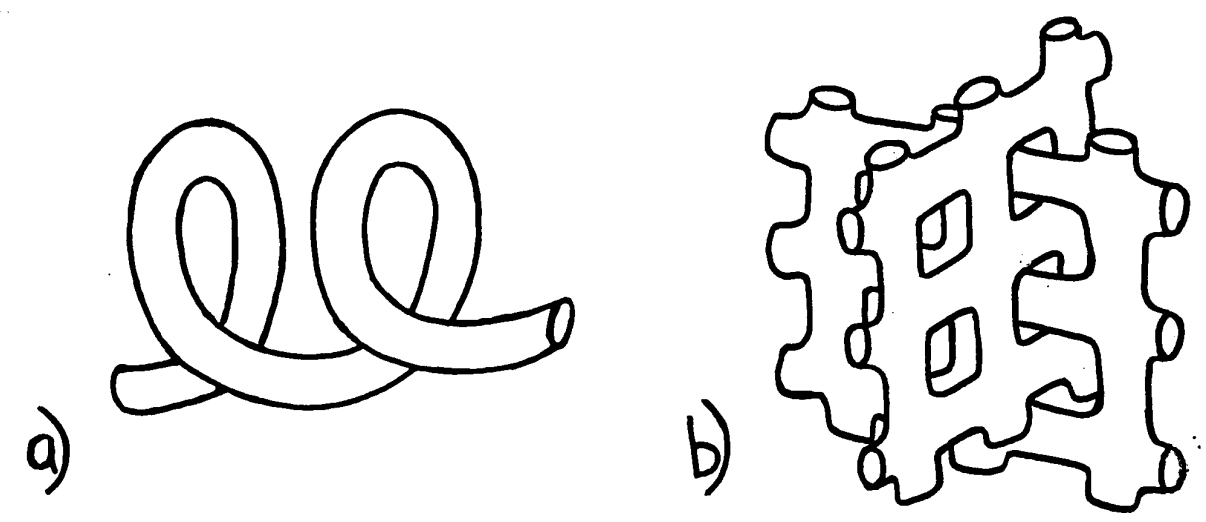

Figure 6: (a) Connected component of Rank 1 having the form of "helix". Open orbits are absent for any direction of $\mathbf{B}$. (b) The example of the Fermi surface of Rank 2 containing two components with different integral directions.

Rank 1 and 2 respectively (see Fig. 6, a,b).

We are going to formulate now the topological theorems concerning the general situation of any complicated Fermi surfaces. We will assume now that the dispersion relation $\epsilon(\mathbf{p})$ is a Morse function on $T^{3}$ and consider the non-singular energy levels $\epsilon(\mathbf{p})=$ const such that $\nabla \epsilon(\mathbf{p}) \neq 0$ everywhere on the corresponding surface. It is easy to see that all the reconstructions of the constant energy surface take place only at the points of singularity. Such the topological type (genus) and the Topological Rank of the constant energy surface are constant on the intervals of regularity. The number of singular constant energy levels is finite for the Morse function $\epsilon(\mathbf{p})$.

The electron trajectories will now be given by the intersections of constant energy surfaces with the planes orthogonal to the magnetic field B. At every plane $\Pi$ orthogonal to $\mathbf{B}$ they can be then considered as the level curves of the quasiperiodic function $\hat{\epsilon}(\mathbf{p})=\left.\epsilon(\mathbf{p})\right|_{\Pi}$ with three quasiperiods. For the case of purely rational directions of $\mathbf{B}$ the corresponding functions become purely periodic. The trajectories can also be represented as the level curves of the height function $h(\mathbf{p})=B_{1} p_{1}+B_{2} p_{2}+B_{3} p_{3}$ restricted on the constant energy levels. This function, however, is not uniquely defined in the three-dimensional torus $T^{3}=R^{3} / \Gamma^{*}$ and becomes the 1 -form in $T^{3}$ after 

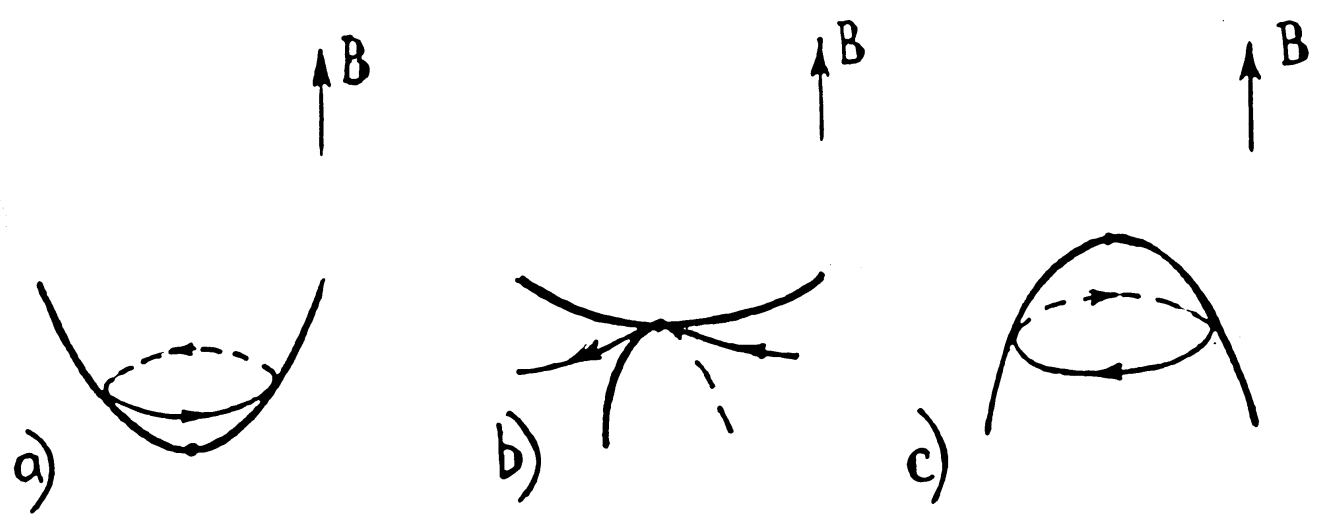

Figure 7: The critical points of the function $\hat{h}(\mathbf{p})$ and the corresponding singular trajectories.

the compactification. The corresponding electron trajectories become then the level curves of the 1-form in $T^{3}$ restricted to the compact smooth energy levels $\epsilon(\mathbf{p})=c$.

We will assume now that the restrictions $\hat{h}(\mathbf{p})$ of $h(\mathbf{p})$ on the constant energy surfaces in $R^{3}$ give also the Morse functions, i.e. all the critical points where $\nabla \epsilon(\mathbf{p}) \| \mathbf{B}$ are non-degenerate on these surfaces.

Definition 7 We call the electron trajectory non-singular if it is not adjacent to the critical point of $\hat{h}(\mathbf{p})$. The trajectories adjacent to critical points of $\hat{h}(\mathbf{p})$ (and the critical points themselves) we will call singular.

According to our assumption there are three types of the critical points of $\hat{h}(\mathbf{p})$ on the constant energy levels. Namely, we can have the local minimum, the saddle point and the local maximum in the $\mathbf{p}$-space (Fig. 7, a-c).

The corresponding pictures in the planes orthogonal to $\mathbf{B}$ are represented on the Fig. 8, a-c.

Let us give also the definitions of "rationality" and "irrationality" of the direction of $\mathbf{B}$.

Definition 8 Let $\left\{\mathbf{g}_{1}, \mathbf{g}_{2}, \mathbf{g}_{3}\right\}$ be the basis of the reciprocal lattice $\Gamma^{*}$. Then: 

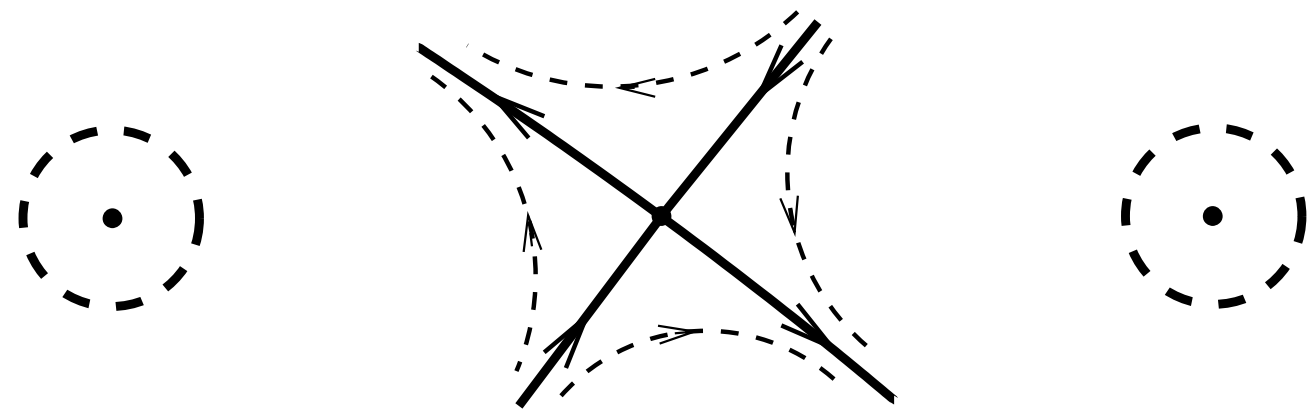

a)

b)

c)

Figure 8: The singular trajectories in the plane orthogonal to $\mathbf{B}$.

1) The direction of $\mathbf{B}$ is rational (or has irrationality 1 ) if the numbers $\left(\mathbf{B}, \mathbf{g}_{1}\right),\left(\mathbf{B}, \mathbf{g}_{2}\right),\left(\mathbf{B}, \mathbf{g}_{3}\right)$ are proportional to each other with rational coefficients.

2) The direction of $\mathbf{B}$ has irrationality 2 if the numbers $\left(\mathbf{B}, \mathbf{g}_{1}\right),\left(\mathbf{B}, \mathbf{g}_{2}\right)$, $\left(\mathbf{B}, \mathbf{g}_{3}\right)$ generate the linear space of dimension 2 over $Q$.

3) The direction of $\mathbf{B}$ has irrationality 3 if the numbers $\left(\mathbf{B}, \mathbf{g}_{1}\right),\left(\mathbf{B}, \mathbf{g}_{2}\right)$, $\left(\mathbf{B}, \mathbf{g}_{3}\right)$ are linearly independent over $Q$.

The conditions (1)-(3) can be formulated also as if the plane $\Pi(\mathbf{B})$ orthogonal to $\mathbf{B}$ contains two linearly independent reciprocal lattice vectors, just one linearly independent reciprocal lattice vector or no reciprocal lattice vectors at all respectively.

It can be seen also that if $\left\{\mathbf{l}_{1}, \mathbf{l}_{2}, \mathbf{l}_{3}\right\}$ is the basis of the original lattice in $\mathbf{x}$-space then the irrationality of the direction of $\mathbf{B}$ will be given by the dimension of the vector space generated by numbers

$$
\left(\mathbf{B}, \mathbf{l}_{2}, \mathbf{l}_{3}\right),\left(\mathrm{B}, \mathbf{l}_{3}, \mathbf{l}_{1}\right),\left(\mathrm{B}, \mathbf{l}_{1}, \mathbf{l}_{2}\right)
$$

over $Q$. Easy to see that these numbers have the meanings of the the magnetic fluxes through the faces of elementary lattice cell. In all our considerations they will always be much smaller than the quantum of magnetic flux and their absolute values will not be important for the quasiclassical pictures. 
However, their ratios having the pure geometrical meanings will play the important role as we will see later.

We are going to consider now the geometry of the non-singular electron trajectories. Let us start with the simplest cases.

1) The Fermi surface has Topological Rank 0.

All the components of the Fermi surface are compact in $R^{3}$ in this case and there is no open trajectories at all.

2) The Fermi surface has Topological Rank 1.

In this case we can have both open and closed electron trajectories. However the open trajectories (if they exist) should be quite simple in this case. They can arise only if the magnetic field is orthogonal to the mean direction of one of the components of Rank 1 and are periodic with the same integer mean direction. There is only the finite number of possible mean directions of open orbits in this case and a finite "net" of one-dimensional curves on the unit sphere giving the directions of $\mathbf{B}$ corresponding to the open orbits. In some special points we can have the trajectories with different mean directions lying in different parallel planes orthogonal to B. Easy to see that in this case the direction of $\mathbf{B}$ should be purely rational such that the orthogonal plane $\Pi(\mathbf{B})$ contains two different reciprocal lattice vectors. It is evident also that there is only the finite number of such directions of $\mathbf{B}$ clearly determined by the mean directions of the components of Rank 1. Let us mention also that the existence of open orbits is not necessary here even for $\mathbf{B}$ orthogonal to the mean direction of some component of Rank 1 as can be seen from the example of the "helix" represented on Fig. 6, a.

3) The Fermi surface has Topological Rank 2.

It can be easily seen that this case gives much more possibilities for the existence of open orbits for different directions of the magnetic field. In particular, this is the first case where the open orbits can exist for the generic direction of $\mathbf{B}$ with irrationality 3. So, in this case we can have the whole regions on the unit sphere such that the open orbits present for any direction of $\mathbf{B}$ belonging to the corresponding region. It is easy to see, however, that the open orbits have also a quite simple description in this case. Namely, any open orbit (if they exist) lies in this case in the straight strip of the finite width for any direction of $\mathbf{B}$ not orthogonal to the integral planes given by the components of Rank 2. The boundaries of the corresponding strips in the planes $\Pi(\mathbf{B})$ orthogonal to $\mathbf{B}$ will be given by the intersection of $\Pi(\mathbf{B})$ with the pairs of integral planes bounding the corresponding components of Rank 
2. It can be also shown ([17], [18]) that every open orbit passes through the strip from $-\infty$ to $+\infty$ and can not turn back.

The contribution of every family of orbits with the same direction to the conductivity coincides in this case with the formula (4) and reveals the strong anisotropy when $\omega_{B} \tau \rightarrow \infty$.

For purely rational directions of $\mathbf{B}$ we can have the situation when the open trajectories with different mean directions present on different components of the Fermi surface. For example, for the "exotic" surface shown at Fig. $6, \mathrm{~b}$ we will have the periodic trajectories along both the $x$ and $y$ directions in different planes orthogonal to $\mathbf{B}$ if $\mathbf{B}$ is directed along the $z$ axis. However, it can be shown that for any direction of $\mathbf{B}$ which is not purely rational this situation is impossible. We have so that for any direction of $\mathbf{B}$ with irrationality 2 or 3 all the open orbits will have the same mean direction and can exist only on the components of Rank 2 with the same (parallel) integral orientation. This statement is a corollary of more general topological theorem which we will discuss in the next part.

At last we note that the directions of $\mathbf{B}$ orthogonal to one of the components of Rank 2 are purely rational and all the non-singular open orbits (if they exist) are rational periodic in this case. For any family of such orbits with the same mean direction the corresponding contribution to the conductivity can then be written in the form (4) in the appropriate coordinate system. However, the direction of open orbits can not be predicted apriori in this case. We will discuss these questions later when consider the "Special rational directions" for the case of arbitrary Fermi surfaces.

Let us discuss now the most general and complicated case of the arbitrary Fermi surface of Topological Rank 3. We describe first the convenient procedure ([23],[28]) of reconstruction of the constant energy surface when the direction of $\mathbf{B}$ is fixed.

Let us fix the direction of $\mathbf{B}$ and consider all closed (in $R^{3}$ ) non-singular electron trajectories on the given energy level. The parts of the constant energy surface covered by the non-singular closed trajectories can be either the tori or the cylinders in $R^{3}$ bounded by the singular trajectories (some of them maybe just points of minimum or maximum) at the bottom and at the top (see Fig. 9).

Let us remove from the Fermi surface the parts containing the compact nonsingular trajectories. The remaining part 


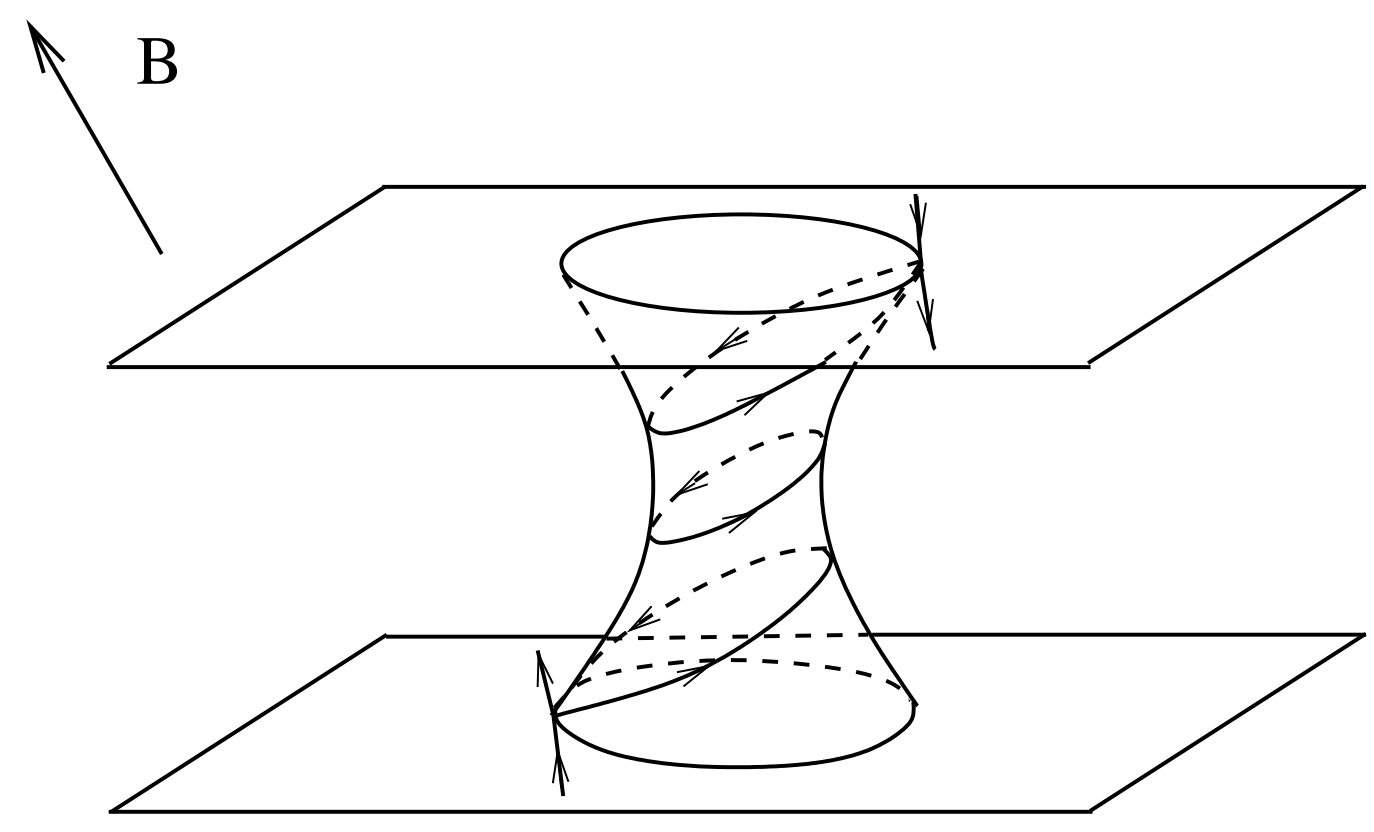

Figure 9: The cylinder of closed trajectories bounded by the singular orbits. (The simplest case of just one critical point on the singular trajectory.)

$$
S_{F} /(\text { Compact Nonsingular Trajectories })=\cup_{j} S_{j}
$$

is a union of the 2-manifolds $S_{j}$ with boundaries $\partial S_{j}$ who are the compact singular trajectories. The generic type is a separatrix orbit with just one critical point like on the Fig. 10.

Definition 9 We call every piece $S_{j}$ the "Carrier of open trajectories". The trajectory is "chaotic" if the genus $g\left(S_{j}\right)$ is greater than 1 . The case $g\left(S_{j}\right)=1$ we call "Topologically Completely Integrable". ${ }^{1}$

Let us fill in the holes by topological $2 D$ discs lying in the planes orthogonal to $\mathbf{B}$ and get the closed surfaces

$$
\bar{S}_{j}=S_{j} \cup(2-d i s c s)
$$

(see Fig. 10).

\footnotetext{
${ }^{1}$ Such systems on $T^{2}$ were discussed for example in [32]; the generic open orbits are topologically equivalent to the straight lines. Ergodic properties of such systems indeed can be nontrivial as it was found by Ya.Sinai and K.Khanin in [33].
} 


\section{Piece consisting of open orbits}

\section{Singular closed \\ orbits}

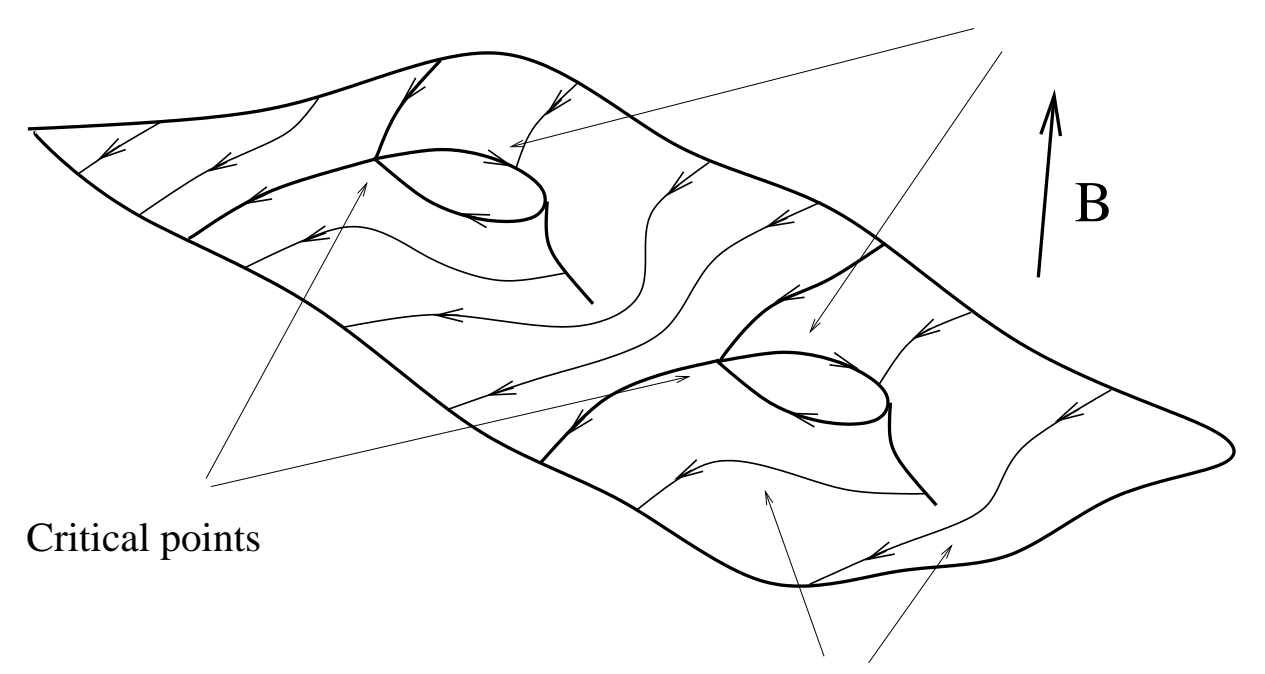

Open orbits

Figure 10: The reconstructed constant energy surface with removed compact orbits and the two-dimensional discs attached to the singular orbits in the generic case of just one critical point on every singular orbit.

This procedure gives again the periodic surface $\bar{S}_{\epsilon}$ after the reconstruction and we can define the "compactified carriers of open trajectories" both in $R^{3}$ and $T^{3}$.

Let us formulate now the main topological theorems concerning the geometry of open trajectories which made a breakthrough in the theory of such dynamical systems on the Fermi surfaces ([16], [19]).

Theorem 1. [16]

Let us fix the energy level $S_{\epsilon}$ and any rational direction $\mathbf{B}_{0}$ such that no two saddle points on $S_{\epsilon}$ are connected in $R^{3}$ by the singular electron trajectory. Then for all the directions of $\mathbf{B}$ close enough to $\mathbf{B}_{0}$ every open trajectory lies in the strip of the finite width between two parallel lines in the plane orthogonal to $\mathbf{B}$.

In fact, the proof of the Theorem 1 was based on the statement that genus of every compactified carrier of open orbits $\bar{S}_{j}$ is equal to 1 in this case.

Theorem 2. [19]

Let a generic dispersion relation 


$$
\epsilon(\mathbf{p}): T^{3} \rightarrow R
$$

be given such that for level $\epsilon(\mathbf{p})=\epsilon_{0}$ the genus $g$ of some carrier of open trajectories $\bar{S}_{i}$ is greater than 1 . Then there exists an open interval $\left(\epsilon_{1}, \epsilon_{2}\right)$ containing $\epsilon_{0}$ such that for all $\epsilon \neq \epsilon_{0}$ in this interval the genus of carrier of open trajectories is less than $g$.

The Theorem 2 claims then that only the "Topologically Completely Integrable case" can be stable with respect to the small variation of energy level and has the generic properties in this situation. For the generic dispersion relations it follows also from the Theorem 1 that this case is the only stable case with respect to the small rotations of the magnetic field $\mathbf{B}$ on the unit sphere $S^{2}$ (see the survey [28] for details).

Physical results of the present authors ([20], [26]).

The very important property of Compactified Carriers of open orbits in the stable case was pointed out by the present authors ([20]) and called later the "Topological Resonance". This property plays the crucial role for the physical phenomena and was first used in [20] (see also [26]) where the "Topological Quantum Numbers" observable in the conductivity were introduced. Namely, consider the stable "Topologically Completely Integrable case" corresponding to genus 1 of the carriers of open trajectories $\bar{S}_{i}$. The "Topological Resonance" dictated by the elementary differential topology claims that all the tori $T^{2}$ represented by $\bar{S}_{i}$ do not intersect each other and have the same (up to the sign) non-divisible homology class in $H_{2}\left(T^{3}\right)$. For the generic (irrationality 3) directions of $\mathbf{B}$ the corresponding coverings of $\bar{S}_{i}$ in $R^{3}$ look like the warped planes with mean directions parallel to the same integral plane in $R^{3}$; the open trajectories in $R^{3}$ have then the same mean directions in the planes orthogonal to $\mathbf{B}$. Our conclusion is that the corresponding contribution of all these trajectories to the conductivity tensor has then the same form (4) in the appropriate coordinate system common for all of them. This fundamental fact leads to existence of measurable characteristics having the topological origin in the conductivity of normal metals.

Let us take a single crystal of a normal metal and consider the full angle diagram of the conductivity $\sigma^{i k}$ for all the directions of $\mathbf{B}$ parameterized by the points of the unit sphere. For the real single-crystal metal only the orbits close to the Fermi surface will give the contribution to the conductivity 
tensor.

Now for all regions where we have just the closed trajectories on the Fermi surface we will have the asymptotic behavior (3) of the conductivity tensor as $B \tau \rightarrow \infty$. The longitudinal conductivity then remains constant in the direction of $\mathbf{B}$ and decreases as $B \rightarrow \infty$ for all the orthogonal directions of electric field. Any other behavior of conductivity tensor shows in this case the presence of open electron trajectories on the Fermi surface lying in the planes orthogonal to B. We know, however, that for any set of open trajectories stable under the rotations of $\mathbf{B}$ we should have the situation described in the Theorems 1,2. The corresponding conductivity tensor $\sigma^{i k}$ is given by the formula (4) in this case and has rank 2 in the limit $B \rightarrow$ $\infty$. We can claim then that any open region on $S^{2}$ with the regular stable behavior of the conductivity different from (3) should correspond to (4) and contain the only one direction $(\eta(\mathbf{B}))$ in the three-dimensional space where the conductivity decreases as $\left(\omega_{B} \tau\right)^{-2}$ as $B \rightarrow \infty$. It can be seen from the previous considerations that this direction should coincide with the mean directions of the open orbits in $\mathbf{p}$-space (let us remind that the projection of the electron trajectory on $x y$-plane in $\mathrm{x}$-space can be obtained by the rotation of the trajectory in $\mathbf{p}$-space by $\pi / 2$ in the plane orthogonal to $\mathbf{B}$ ). We can extract now from Theorems 1, 2 that $\eta(\mathbf{B})$ should always belong to some integral plane $\Gamma_{\alpha}$ (with respect to reciprocal lattice) which is the same for the whole stability region on the unit sphere and represents the homology class $c \in \mathrm{H}_{2}\left(T^{3}\right)$ of the stable two-dimensional tori $T_{i}^{2}$. The stability of this plane w.r.t. the small rotations of the magnetic field gives then the easy possibility to get this characteristic in the experiment.

The total set of the stability regions $\Omega_{\alpha}$ on the unit sphere with the corresponding integral planes $\Gamma_{\alpha}$ was called in [20]) the "Topological Quantum characteristics" of the normal metal. These quantities have the quantum origin being obtained from the apriori unknown dispersion relation $\epsilon(\mathbf{p})$ but appear in a purely geometrical way from the geometry of the Fermi surface.

The corresponding integral planes $\Gamma_{\alpha}$ can then be given by three integer numbers $\left(n_{\alpha}^{1}, n_{\alpha}^{2}, n_{\alpha}^{3}\right)$ (up to the common multiplier) from the equation

$$
n_{\alpha}^{1}[\mathbf{x}]_{1}+n_{\alpha}^{2}[\mathbf{x}]_{2}+n_{\alpha}^{3}[\mathbf{x}]_{3}=0
$$

where $[\mathbf{x}]_{i}$ are the coordinates in the basis $\left\{\mathbf{g}_{1}, \mathbf{g}_{2}, \mathbf{g}_{3}\right\}$ of the reciprocal lattice, or equivalently 


$$
n_{\alpha}^{1}\left(\mathbf{x}, \mathbf{l}_{1}\right)+n_{\alpha}^{2}\left(\mathbf{x}, \mathbf{l}_{2}\right)+n_{\alpha}^{3}\left(\mathbf{x}, \mathbf{l}_{3}\right)=0
$$

where $\left\{\mathbf{l}_{1}, \mathbf{l}_{2}, \mathbf{l}_{3}\right\}$ is the basis of the initial lattice in the coordinate space.

The numbers $\left(n_{\alpha}^{1}, n_{\alpha}^{2}, n_{\alpha}^{3}\right)$ were called the "Topological Quantum numbers" of a dispersion relation in metal.

Let us add also that the number of tori $T_{i}^{2}$ being even can still be different for the different points of stability zone $\Omega_{\alpha}$. We can then introduce in the general situation the "sub-boundaries" of the stability zone which are the piecewise smooth curves inside $\Omega_{\alpha}$ where the number of tori generically changes by 2 . The asymptotic behavior of conductivity will still be described by the formula (4) in this case but the dimensionless coefficients will then "jump" on the sub-boundaries of stability zone. Let us however mention here that this situation can be observed only for rather complicated Fermi surfaces.

As was first shown by S.P.Tsarev ([29]) the more complicated chaotic open orbits can still exist on rather complicated Fermi surfaces $S_{F}$. Such, the example of open trajectory which does not lie in any finite strip of finite width was constructed. The corresponding direction of $\mathbf{B}$ had the irrationality 2 in this example and the closure of the open orbit was a "half" of the surface of genus 3 separated by the singular closed trajectory non-homotopic to zero in $T^{3}$. However, the trajectory had in this case the asymptotic direction even not being restricted by any straight strip of finite width in the plane orthogonal to $\mathbf{B}$.

As was shown later in [23], [22] this situation always takes place for any chaotic trajectory for the directions of $\mathbf{B}$ with irrationality 2. We have so, that for non-generic "partly rational" directions of $\mathbf{B}$ the chaotic behavior is still not "very complicated" and resembles some features of stable open electron trajectories.

The corresponding asymptotic behavior of conductivity should reveal also the strong anisotropy properties in the plane orthogonal to $\mathbf{B}$ although the exact form of $\sigma^{i k}$ will be slightly different from (4) for this type of trajectories. By the same reason, the asymptotic direction of orbit can be measured experimentally in this case as the direction of lowest longitudinal conductivity in $R^{3}$ according to kinetic theory. The measure of the corresponding set on the unit sphere is obviously zero for such type of trajectories being restricted by the measure of directions of irrationality 2 . 
The more complicated examples of chaotic open orbits were constructed in [23] for the Fermi surface having genus 3. The direction of the magnetic field has the irrationality 3 in this case and the closure of the chaotic trajectory covers the whole Fermi surface in $T^{3}$. These types of the open orbits do not have any asymptotic direction in the planes orthogonal to $\mathbf{B}$ and have rather complicated form "walking everywhere" in these planes. Let us discuss later this case in more details.

The recent topological results ([23], [28]).

After the works [16], [19], [20] a systematic investigation of open orbits was completed by I.A.Dynnikov (see [21]-[23], [28]). In particular the total picture of different types of the open orbits for generic dispersion relations was presented $([28])$. Let us describe here the corresponding topological results.

Theorem 3 ([23], [28]).

Let us fix the dispersion relation $\epsilon=\epsilon(\mathbf{p})$ and the direction of $\mathbf{B}$ of irrationality 3 and consider all the energy levels for $\epsilon_{\min } \leq \epsilon \leq \epsilon_{\max }$. Then:

1) The open electron trajectories exist for all the energy values $\epsilon$ belonging to the closed connected energy interval $\epsilon_{1}(\mathbf{B}) \leq \epsilon \leq \epsilon_{2}(\mathbf{B})$ which can degenerate to just one energy level $\epsilon_{1}(\mathbf{B})=\epsilon_{2}(\mathbf{B})=\epsilon_{0}(\mathbf{B})$.

2) For the case of the non-degenerate energy interval the set of compactified carriers of open trajectories $\bar{S}_{\epsilon}$ is always a disjoint union of twodimensional tori $T^{2}$ in $T^{3}$ for all $\epsilon_{1}(\mathbf{B}) \leq \epsilon \leq \epsilon_{2}(\mathbf{B})$. All the tori $T^{2}$ for all the energy levels do not intersect each other and have the same (up to the sign) indivisible homology class $c \in H_{2}\left(T^{3}\right), c \neq 0$. The number of tori $T^{2}$ is even for every fixed energy level and the corresponding covering $\bar{S}_{\epsilon}$ in $R^{3}$ is a locally stable family of parallel ("warped") integral planes $\Pi_{i}^{2} \subset R^{3}$ with common direction given by $c$. The form of $\bar{S}_{\epsilon}$ described above is locally stable with the same homology class $c \in H_{2}\left(T^{3}\right)$ under small rotations of $\mathbf{B}$. All the open electron trajectories at all the energy levels lie in the strips of finite width with the same direction and pass through them. The mean direction of the trajectories is given by the intersections of planes $\Pi(\mathbf{B})$ with the integral family $\Pi_{i}^{2}$ for the corresponding "stability zone" on the unit sphere.

3) The functions $\epsilon_{1}(\mathbf{B}), \epsilon_{2}(\mathbf{B})$ defined for the directions of $\mathbf{B}$ of irrationality 3 can be continuated on the unit sphere $S^{2}$ as the piecewise smooth functions such that $\epsilon_{1}(\mathbf{B}) \geq \epsilon_{2}(\mathbf{B})$ everywhere on the unit sphere.

4) For the case of trivial energy interval $\epsilon_{1}=\epsilon_{2}=\epsilon_{0}$ the corresponding open trajectories may be chaotic. Carrier of the chaotic open trajectory is 
homologous to zero in $H_{2}\left(T^{3}, Z\right)$ and has genus $\geq 3$. For the generic energy level $\epsilon=\epsilon_{0}$ corresponding directions of magnetic fields $\xi \in S^{2}$ leading to the chaotic trajectories belong to the countable union of the codimension 1 subsets. Therefore a measure of this set is equal to zero on $S^{2}$.

The whole manifold $\bar{S}_{\epsilon}$ is always homologous to zero in $T^{3}$ and all the two dimensional tori $T^{2}$ can be always divided in two equal groups $\left\{T_{i+}^{2}\right\}$, $\left\{T_{i-}^{2}\right\}$ according to the direction of the electron motion. As can be proved using Theorem 1 ([28]) the "stability zones" form the everywhere dense set on the unit sphere for the generic dispersion relations. All the non-closed trajectories stable under the small rotations of $\mathbf{B}$ should have thus the form described above.

All the trajectories described in Theorem 3 give the same form of conductivity (4) as $B \rightarrow \infty$ in the appropriate coordinate system (Topological resonance).

Let us consider now the non-generic cases of the magnetic fields of irrationality 1 and 2 . The Theorem 3 should be slightly modified in this case but has the same main features as in the case of fully irrational magnetic field ([28]). Namely, the set of carriers of open trajectories $\bar{S}_{\epsilon}$ can contain now the two-dimensional tori $T_{s}^{2 \prime}$ having the zero homology class in $H_{2}\left(T^{3}\right)$ in addition to the family of parallel tori with non-zero homology classes described above. The corresponding covering of these components in $R^{3}$ are "warped" periodic cylinders and all the open trajectories belonging to these components are purely periodic. As it is easy to see these components of $\bar{S}_{\epsilon}$ are stable with respect to the small rotations of $\mathbf{B}$ in the plane orthogonal to the axis of cylinder and disappear after any other small rotation. The part (1) of the Theorem 2 will be true also for rational or "partly rational" directions of $\mathbf{B}$ with some connected energy interval $\epsilon_{1}^{\prime}(\mathbf{B}) \leq \epsilon \leq \epsilon_{2}^{\prime}(\mathbf{B})$. However, the boundary values $\epsilon_{1}^{\prime}(\mathbf{B}), \epsilon_{2}^{\prime}(\mathbf{B})$ do not necessarily coincide in this case with the values of piecewise smooth functions $\epsilon_{1}(\mathbf{B}), \epsilon_{2}(\mathbf{B})$ defined everywhere on $S^{2}$ according to Theorem 3 ([28]). Namely, we will have instead the relations $\epsilon_{1}^{\prime}(\mathbf{B}) \leq \epsilon_{1}(\mathbf{B}) \leq \epsilon_{2}(\mathbf{B}) \leq \epsilon_{2}^{\prime}(\mathbf{B})$ for all such directions of $\mathbf{B}$ where all the components of $\bar{S}_{\epsilon}$ belonging to intervals $\left[\epsilon_{1}^{\prime}(\mathbf{B}), \epsilon_{1}(\mathbf{B})\right),\left(\epsilon_{2}(\mathbf{B}), \epsilon_{2}^{\prime}(\mathbf{B})\right]$ consist of the tori homologous to zero in $T^{3}$. As we will see this cases can be observed experimentally for those $\mathbf{B}$ where the Fermi level lies in the one of such intervals and only the "partly stable" non-closed trajectories exist on the Fermi surface.

The chaotic open orbits can exist also for the directions of $\mathbf{B}$ of irrational- 
ity 2 (Tsarev chaotic orbits). Their behavior however reveals completely different properties from the case of generic directions of magnetic field as we already discussed above.

The "partly-stable" cylinders described above do not intersect the "absolutely stable" components of $\bar{S}_{\epsilon}$ and all the open trajectories will still have the same mean direction if $\mathbf{B}$ is not orthogonal to corresponding integral plane $\Gamma_{\alpha}$ (let us mention that all the trajectories lying on "regular" parallel integral planes in $\bar{S}_{\epsilon} \subset R^{3}$ will be also periodic with the same period in this case). The form of the conductivity tensor will still be described by the formula (4) but the numerical values of dimensionless coefficients will jump for those non-generic directions of $\mathbf{B}$ where the situation described appears. In particular, the asymptotic behavior (4) will arise on the one-dimensional curves for those directions of $\mathbf{B}$ where $\epsilon_{F} \in\left[\epsilon_{1}^{\prime}(\mathbf{B}), \epsilon_{1}(\mathbf{B})\right) \cup\left(\epsilon_{2}(\mathbf{B}), \epsilon_{2}^{\prime}(\mathbf{B})\right]$ being stable only for rotations of $\mathbf{B}$ in the corresponding direction. As follows from the statements above the corresponding directions of $\mathbf{B}$ can have at most the irrationality 2 and the corresponding one-dimensional curves are always the parts of the circles orthogonal to some integer vector in the reciprocal lattice $\Gamma^{*}$.

Let us make now a special remark about the "Special directions" of B orthogonal to the integral planes $\Gamma_{\alpha}$ if this direction belongs to the corresponding stability zone $\Omega_{\alpha}$. The direction of $\mathbf{B}$ is then purely rational and all the corresponding open orbits (if they exist) should be periodic in $R^{3}$. However, the mean directions of these open orbits can be different in this case for the different planes orthogonal to the magnetic field and the corresponding contributions to conductivity can not be then written in the form (4) in the same coordinate system. The conductivity tensor $\sigma^{i k}$ can have then the full rank in the limit $B \tau \rightarrow \infty$ and the conductivity remains constant for all the directions in $R^{3}$ in the strong magnetic field limit. This situation, however, is completely unstable and disappear after any small rotation of $\mathbf{B}$.

The second possibility in the case of such directions is that all the open trajectories become singular and form the "singular periodic nets" in the planes orthogonal to $\mathbf{B}$ (see Fig. 11). The asymptotic behavior of conductivity is described then by formula (3) but is also completely unstable and changes to (4) after any small rotation of $\mathbf{B}$.

Let us add that the same situations can arise also on the stable twodimensional tori $T_{i}^{2}$ in this case where the directions of open orbits can not be defined anymore as the intersection of $\Gamma_{\alpha}$ with the plane $\Pi(\mathbf{B})$. Such, we 


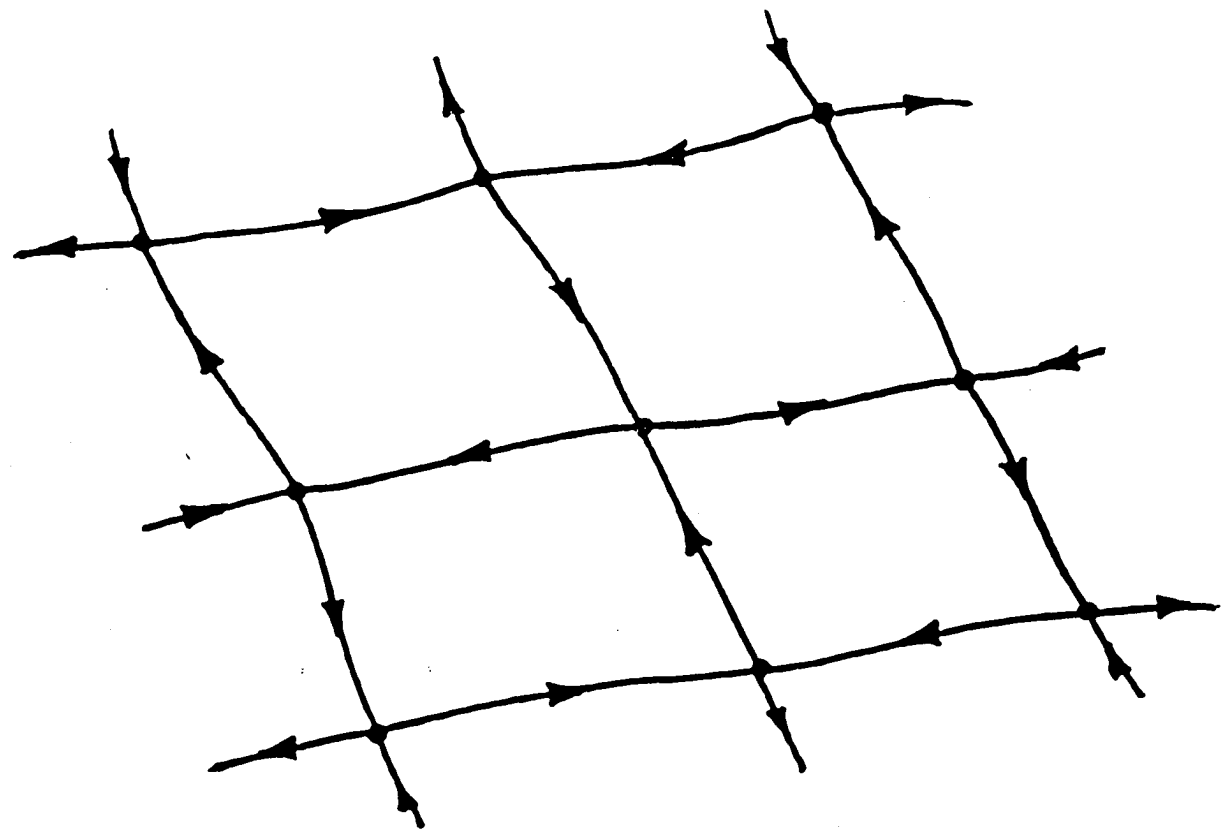

Figure 11: The "singular periodic net" of open trajectories in the plane orthogonal to $\mathbf{B}$.

can have either the "singular nets" or the regular periodic open orbits on these tori for this special direction. Also we can have the open orbits with different mean directions on different tori in this case but the number of such tori should be $\geq 4$ for any physical type of dispersion relations. This situation can thus also be observed only for rather complicated Fermi surfaces.

Let us mention also that for the directions of $\mathbf{B}$ close to this special rational directions the widths of the straight strips containing the regular open orbit can become very big in the planes $\Pi(\mathbf{B})$ (see Fig. 12). So from physical point of view the conductivity phenomena will not "feel" the mean directions of open orbits for the directions of $\mathbf{B}$ close enough to these special points even for rather big (but finite) values of magnetic field. Instead, the oscillations of the trajectory within the strip (Fig. 12) will be essential for conductivity up to the rather big values of $B$ such that $\omega_{B} \tau \sim L / p_{0}$ (where $L$ is the width of the strip and $p_{0}$ is the size of the Brilluone zone). These situation exists, however, if the open orbits with corresponding direction exist also for $\mathbf{B}=\mathbf{B}_{0}$ where $\mathbf{B}_{0}$ is the special rational direction in the stability zone. For $\mathbf{B}$ close to $\mathbf{B}_{0}$ we will observe then exactly this direction up to the values of $B$ such that $\omega_{B} \tau \sim L / p_{0}$ and then the regime will change to the common situation corresponding to given stability zone. Experimentally we will observe then the "small spots" around these directions on the unit 


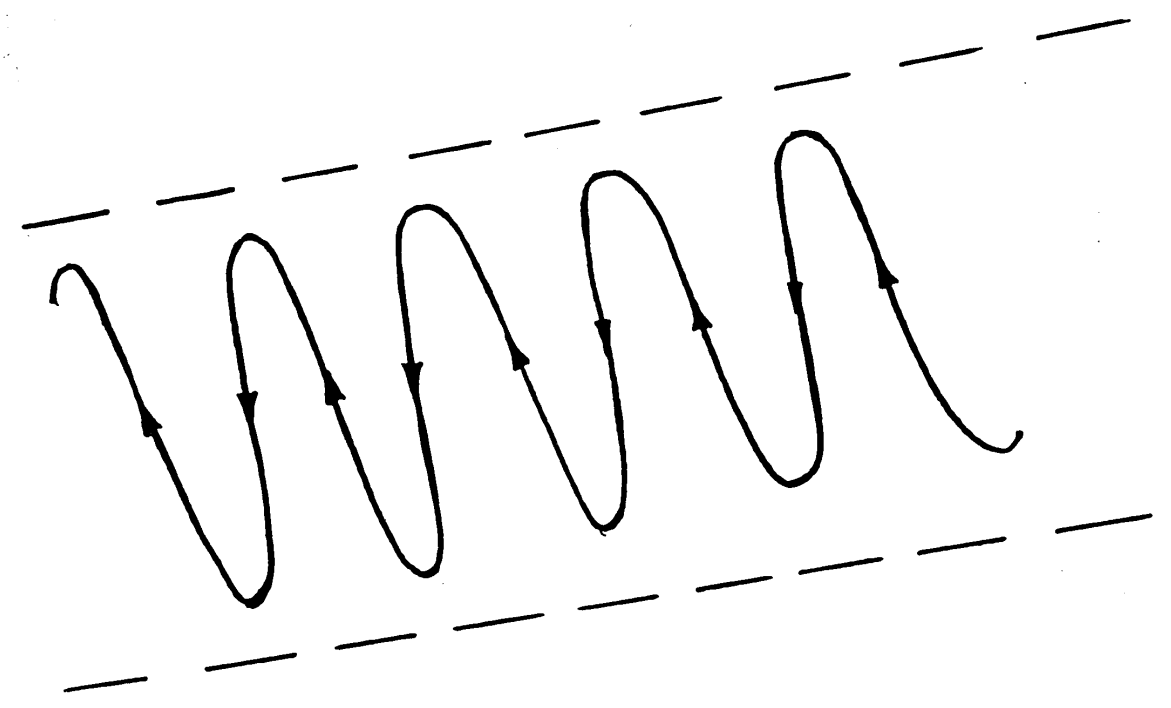

Figure 12: The wide finite strip in the plane $\Pi(\mathbf{B})$ containing the open orbits for B close to "Special rational direction" within the stability zone.

sphere where the anisotropy of $\sigma^{i k}$ corresponds to the direction of orbits for $\mathbf{B}=\mathbf{B}_{0}$. In the most complicated case when we have the open orbits with different mean directions for $\mathbf{B}=\mathbf{B}_{0}$ we will have then the finite conductivity for all the directions in $R^{3}$ in the corresponding spot for rather big values of $B$.

For the "special rational directions" corresponding to the "singular net" on the stable tori $T_{i}^{2}$ the behavior of $\sigma^{i k}$ will correspond to the common form for a given stability zone even for $\mathbf{B}$ very close to $\mathbf{B}_{0}$. However, the measure of open orbits will tend to zero as $\mathbf{B} \rightarrow \mathbf{B}_{0}$ in the stability zone. The dimensionless coefficients (*) in the formula (4) will vanish then for $\mathbf{B} \rightarrow \mathbf{B}_{0}$ although the integral plane $\Gamma_{\alpha}$ will be observable up to $\mathbf{B}=\mathbf{B}_{0}$.

We mention here at last that both cases when $\mathbf{B}_{0}^{\alpha}$ belongs or does not belong to the corresponding stability zone $\Omega_{\alpha}$ are possible in the examples.

Let us describe also the situation on the boundary of the stability zone on the unit sphere. We will consider the simplest case of the Fermi surface on Fig. 5,c but the same situation will take place also for general case. Let us consider the sections of this Fermi surface by planes orthogonal to $\mathbf{B}$ where direction of $\mathbf{B}$ is close to normal direction of the warped planes (see Fig. 9, a).

We have then the two parallel planes (with holes) in $\mathbf{p}$-space corresponding to two tori $T^{2}$ in $T^{3}$ divided by cylinder of closed trajectories. The set 


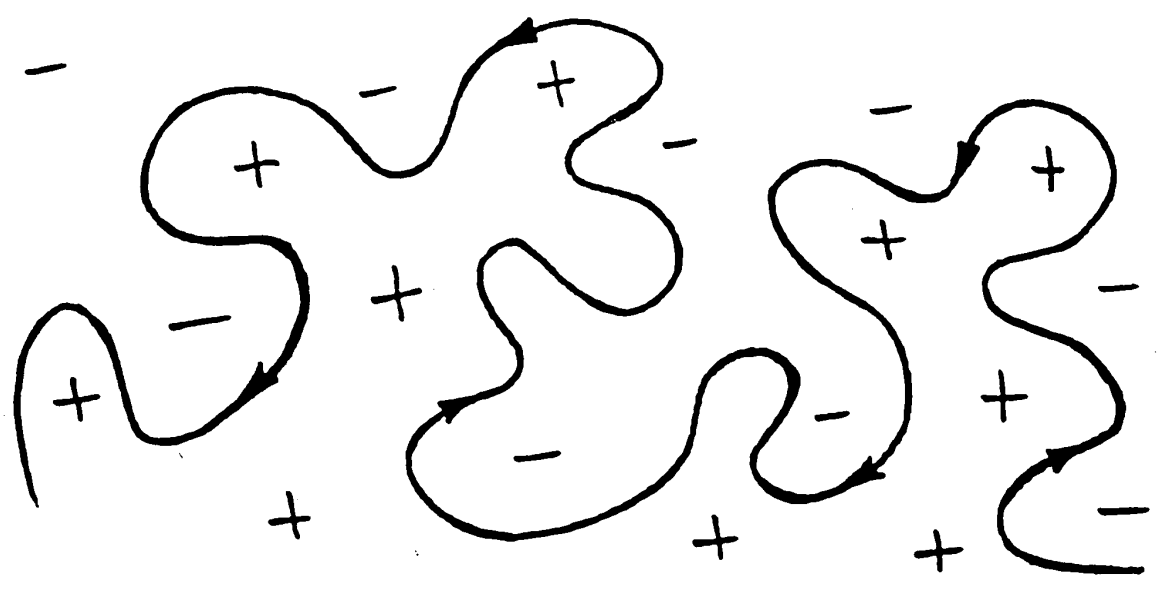

Figure 13: The chaotic open orbit of Dynnikov type. The signs " + " and " - " denote the regions with $\epsilon>\epsilon_{F}$ and $\epsilon<\epsilon_{F}$ respectively.

of compactified carriers of open orbits $\bar{S}_{F}$ on the Fermi level is just the pair of parallel tori in this case and the height of the cylinder of closed trajectories depends on the direction of magnetic field B. For the directions of B belonging to the boundary of the stability zone this height is zero and the corresponding cylinder becomes just the singular orbit connecting two critical points. For the directions of $\mathbf{B}$ far enough from the normal direction the trajectories can "jump" from one plane to another and the stable open orbits will be destroyed. This case describes well the general situation on the boundary of stability zone for generic Fermi surfaces. Namely, we have generically two tori covered by the open orbits divided by cylinder of closed trajectories near the boundary of the stability zone. The height of the cylinder becomes equal to zero at the boundary of the stability zone and then the tori disappear giving the new reconstruction of Fermi surface $\bar{S}_{F}$. The phase space occupied by the open orbits thus remains constant near the boundary curves and the corresponding conductivity tensor (4) has the finite values of coefficients $*$ up to the boundary of the stability zone.

Let us discuss now in more details the situation of "chaotic open" electron orbits which can appear for the cases of $\mathbf{B}$ with irrationality 3 ([23]). The approximate form of trajectories of such kind is represented on Fig. 13.

For the special examples constructed in [23] it can be shown $([27])$ that 
there exists a special direction in $\Pi(\mathbf{B})$ in which the "amplitude of oscillations" of trajectories grows faster than in the orthogonal direction. However, in the general situation this fact is not strictly proved until now.

It can be proved ([28]) that the set of directions of $\mathbf{B}$ corresponding to such kind of trajectories has the measure zero on $S^{2}$ if we restrict ourselves on the fixed generic Fermi level $\epsilon(\mathbf{p})=\epsilon_{F}$. This means actually that the experimentally observable set of such directions of $\mathbf{B}$ has generically measure zero for the case of normal metals though they still can be found for some special directions.

Nevertheless, the total set of such directions for the whole dispersion relation has a very complicated (fractal type) structure and its total measure is still unknown in the general situation.

Conjecture. (S.P.Novikov).

The total set of the directions of $\mathbf{B}$ corresponding to the chaotic behavior has the measure 0 for the whole generic dispersion relation and the Hausdorf dimension strictly less than 2 .

The contribution to the conductivity tensor for the type of chaotic trajectories described above is rather different from both the cases (3) and (4) ([25]). Let us mention first of all that the component of $\bar{S}_{F}$ on the Fermi level carrying the trajectory of this kind should have at least genus 3 . This means for instance that for any Fermi surface $S_{F}$ with the genus less than 6 we can have only one such component (homologous to zero) in the three-dimensional torus $T^{3}$. For any physical dispersion relation $(\epsilon(\mathbf{p})=\epsilon(-\mathbf{p}))$ we will have then that this component should be invariant w.r.t. to the inversion transformation $\mathbf{p} \rightarrow-\mathbf{p}$ for the appropriate initial point $\mathbf{p}_{0}=0$. It follows from this symmetry that the average of any component of group velocity $\mathbf{v}_{g r}$ over this part of the Fermi surface should be zero for any physical Fermi surface of genus $\leq 6$. The fact that the chaotic trajectory is everywhere dense on this component leads then to the fact that the contribution to the conductivity tensor disappears as $B \tau \rightarrow \infty$ for such type of trajectories in all the directions in $R^{3}$ including the direction of magnetic field $\mathbf{B}$. The asymptotic form of $\sigma^{i k}$ as $B \tau \rightarrow \infty$ was suggested in [25] and contains the fractional powers of parameter $\omega_{B} \tau$ decreasing as $B \rightarrow \infty$. Let us just write here the corresponding form in the coordinates with $\mathbf{B}$ directed along the $z$ axis and the $x$ and $y$ axes oriented in a special way connected with some aspects of 
geometry of the chaotic orbit:

$$
\sigma^{i k} \simeq \frac{n e^{2} \tau}{m^{*}}\left(\begin{array}{ccc}
\left(\omega_{B} \tau\right)^{-2 \alpha} & \left(\omega_{B} \tau\right)^{-1} & \left(\omega_{B} \tau\right)^{-\alpha-\gamma} \\
\left(\omega_{B} \tau\right)^{-1} & \left(\omega_{B} \tau\right)^{-2 \beta} & \left(\omega_{B} \tau\right)^{-\beta-\gamma} \\
\left(\omega_{B} \tau\right)^{-\alpha-\gamma} & \left(\omega_{B} \tau\right)^{-\beta-\gamma} & \left(\omega_{B} \tau\right)^{-2 \gamma}+T^{2} / \epsilon_{F}^{2}
\end{array}\right)
$$

where $0<\alpha, \beta, \gamma<1, \alpha+\beta=1$ and $T / \epsilon_{F}$ is a parameter of order of $10^{-4}$. The contribution (6) should be added with the contribution of the closed orbits having the form (3). The conductivity along the magnetic field $\mathbf{B}$ will then be finite at $\omega_{B} \tau \rightarrow \infty$ due to the presence of closed trajectories. However, this conductivity should have the local minimum on the angle diagram in this case since the finite part of the Fermi surface will be excluded from it in the strong magnetic field limit.

We can describe now the total picture for the angle diagram of conductivity in normal metal in the case of geometric strong magnetic field limit. Namely, we can observe the following objects on the unit sphere parameterizing the directions of $\mathbf{B}$ :

1) The "stability zones" $\Omega_{\alpha}$ corresponding to some integral planes $\Gamma_{\alpha}$ in the reciprocal lattice ("Topological Quantum numbers"). This "Topological Type" of open trajectories is stable with respect to small rotations of $\mathbf{B}$ and this is the only open orbits regime which can have a non-zero measure on the unit sphere. All the "stability zones" have the piecewise smooth boundaries on $S^{2}$ and are given by the condition

$$
\epsilon_{1}(\mathbf{B}) \leq \epsilon_{F} \leq \epsilon_{2}(\mathbf{B})
$$

where $\epsilon_{1}(\mathbf{B}), \epsilon_{2}(\mathbf{B})$ are the piecewise smooth functions defined in the Theorem 3. Generally speaking, this set is not everywhere dense anymore being just a subset of the corresponding set for the whole dispersion relation and have some rather complicated geometry on the unit sphere.

The corresponding behavior of conductivity is described by the formula (4) and reveals the strong anisotropy in the planes orthogonal to the magnetic field. For rather complicated Fermi surfaces we can observe also the "subboundaries" of the stability zones where the coefficients in (4) have the sharp "jump". 
2) The net of the one-dimensional curves containing directions of irrationality $\leq 2$ where the additional two-dimensional tori (homologous to zero in $T^{3}$ ) can appear. The corresponding parts of the net are always the parts of the big (passing through the center of $S^{2}$ ) circles orthogonal to some reciprocal lattice vector where the condition

$$
\epsilon_{1}^{\prime}(\mathbf{B}) \leq \epsilon_{F} \leq \epsilon_{2}^{\prime}(\mathbf{B})
$$

is satisfied. The asymptotic behavior of conductivity is given again by the formula (4).

Let note also that these special curves on $S^{2}$ can be considered actually as the reminiscent of the bigger stability zones if we don't restrict ourselves just by one Fermi surface. The structure of such sets thus can be used to get more information about the corresponding total structure for the whole dispersion relation in metal. In particular, the mean direction of such open orbits coincides with the mean direction of the generic open orbits in the intersections of the net with the stability zones (except the "Special rational directions"). The corresponding conductivity tensor is given then by the same formula (4) where the dimensionless coefficients "jump" on the curves of the net.

3) The "Special rational directions".

Let us remind that we call the special rational direction the direction of B orthogonal to some plane $\Gamma_{\alpha}$ in case when this direction belongs to the same stability zone on the unit sphere. We can have here all the possibilities described earlier for this situation (i.e. regular behavior with vanishing coefficients in (4), spots with isotropic or anisotropic behavior of conductivity different from the given by corresponding "Topological quantum numbers", "partly stable" isotropic or anisotropic addition to the (4), etc.)

4) The chaotic open orbits of Tsarev type ( $\mathbf{B}$ of irrationality 2).

We can have points on the unit sphere where the open orbits are chaotic in Tsarev sence. All open trajectories still have the asymptotic direction in this case and the conductivity reveals the strong anisotropy in the plane orthogonal to $\mathbf{B}$ as $B \rightarrow \infty$. The $B$ dependence, however can be slightly different from the formula (4) in this case. 
5) The chaotic open orbits of Dynnikov type ( $\mathbf{B}$ of irrationality 3 ).

For some points on $S^{2}$ we can have the chaotic open orbits of Dynnikov type on the Fermi surface. At these points the local minimum of conductivity along the magnetic field is expected. The conductivity along $\mathbf{B}$ however remains finite as $B \rightarrow \infty$ in general situation because of the contribution of closed trajectories. The main asymptotic of conductivity tensor in plane orthogonal to B can then be extracted from formula (6) in strong magnetic field limit.

6) At last we can have the open regions on the unit sphere where only the closed trajectories on the Fermi level are present. The asymptotic behavior of conductivity tensor is given then by the formula (3).

Let now point out some new features connected with the "magnetic breakdown" (self-intersecting Fermi surfaces) which can be observed for rather strong magnetic fields. Up to this point it has been assumed throughout that different parts of the Fermi surface do not intersect with each other. However, it is possible for some special lattices that the different components of the Fermi surface (parts corresponding to different conductivity bands) come very close to each other and may have an effective "reconstruction" as a result of the "magnetic breakdown" in strong magnetic field limit. In this case we can have the situation of the electron motion on the self-intersecting Fermi surface such that the intersections with other pieces do not affect at all the motion on one component. (The physical conditions for the corresponding values of $B$ can be found in [10]). In this case the picture described above should be considered independently for all the non-selfintersecting pieces of Fermi surface and we can have simultaneously several independent angle diagrams of this form on the unit sphere. Such we can have here the overlapping stability zones where the open orbits can have different mean directions. The correspondent conductivity tensor will then be given just as a sum of all conductivity tensors corresponding to different non-selfintersecting components. (The problem of the magnetic breakdown was brought to the authors' attention by M.I.Kaganov.)

Let us mention also that in the paper [24] the possibilities of the investigation of total topological characteristics of whole dispersion relation $\epsilon(\mathbf{p})$ were discussed. In particular the behavior of electrons injected in the empty band of semiconductor in the presence of magnetic field was considered. How- 
ever, the corresponding magnetic fields should be extremely high $\left(\sim 10^{2} t\right)$ in this case which make such experiments very difficult. Most probably this situation should be considered now just as theoretical possibility.

Coming back to the old results we can see now that statement of I.M.Lifshitz an V.G.Peschanski concerning the "thin spatial net" (Fig. 3, a,b) can be considered as the particular case of general topological theorem with the simplest set of planes $\Gamma_{1}, \Gamma_{2}, \Gamma_{3}$ given by the coordinate planes $x y, y z$ and $x z$ (i.e. types $( \pm 1,0,0),(0, \pm 1,0),(0,0, \pm 1)$ only). We predict the existence of the integral planes also in the case of analytic dispersion relation (5) for all the stability zones shown on Fig. 4. As then follows from the rotational symmetry of the Fermi surfaces the integral planes $\Gamma_{i}$ in this case should be orthogonal to the unit vectors directed to the centers of zones shown at Fig. 4 from the center of unit sphere. This situation, however, is not necessary in the general case and caused here by the symmetry of the dispersion relation for the biggest stability zones. All centers of zones at Fig. 4 are the "Special rational directions" of the magnetic field in this case. As again follows from rotational symmetry the corresponding structure of orbits can be only the "singular nets" in the planes orthogonal to $\mathbf{B}_{0}$. The conductivity tensor should thus correspond to the form (4) with the same "Topological quantum numbers" everywhere and the vanishing dimensionless coefficients $*$ near the centers of zones. The one-dimensional curves on the Fig. 4 correspond in this case to the non-generic directions of $\mathbf{B}$ with the "partly stable" periodic open trajectories.

As we have already mentioned we expect here also the infinite number of smaller stability zones (as well as the chaotic regimes) with the infinite number of corresponding integral planes $\Gamma_{\alpha}$. The geometry of the zones and the corresponding integer numbers $\left(n_{1}^{\alpha}, n_{2}^{\alpha}, n_{3}^{\alpha}\right)$ will depend on parameters $\alpha$, $\beta, \delta$ and $\zeta_{0}$ in (5) being defined by geometry of the correspondent Fermi surface. The smaller stability zones correspond in the general picture to bigger numbers $\left(n_{1}^{\alpha}, n_{2}^{\alpha}, n_{3}^{\alpha}\right)$ and are more sensitive to the change of parameters in the analytic approximation.

The early experimental investigations of different conductivity behavior in strong magnetic fields can be found in [4]-[7]. Let us consider at the end the experimental results for conductivity in the plane orthogonal to $\mathbf{B}$ for Au obtained by Yu.P.Gaidukov in 1959 ([7]).

For the comparison with the experimental data we give here the asymp- 
totic form of the resistance tensor, inverse to $\sigma: R=\sigma^{-1}$ in the same basis as $\sigma$ above (see [10], [11]).

Case 1. (Closed orbits).

$$
\hat{R} \simeq \frac{m^{*}}{n e^{2} \tau}\left(\begin{array}{ccc}
1 & \omega_{B} \tau & 1 \\
\omega_{B} \tau & 1 & 1 \\
1 & 1 & 1
\end{array}\right)
$$

(Part of matrix proportional to $B$ is skew-symmetrical).

Case 2. Generic open orbits.

$$
\hat{R} \simeq \frac{m^{*}}{n e^{2} \tau}\left(\begin{array}{ccc}
\left(\omega_{B} \tau\right)^{2} & \omega_{B} \tau & \omega_{B} \tau \\
\omega_{B} \tau & 1 & 1 \\
\omega_{B} \tau & 1 & 1
\end{array}\right)
$$

As we can see from (8), we should observe $B^{2}$ - dependence of the resistance $\rho \sim\left(B^{2} \cos ^{2} \alpha\right) \rho_{0}$ in the plane orthogonal to $\mathbf{B}$ in the Case 2 . The coefficient $\left(\cos ^{2} \alpha\right)$ is equal to 1 for the electric field directed along the vector $\mathbf{e}_{1}$ - zero eigenvector for conductivity tensor (4) in the plane orthogonal to B. The same behavior will also be qualitatively true for the case of Tsarev chaotic open orbits with slightly different asymptotic in $B$.

Let us omit here the expression for resistivity tensor corresponding to more general Dynnikov chaotic trajectories ( [25]) and write here just the expression for resistivity in the plane orthogonal to B. According to the conjecture in [25] we should have the "scaling behavior" of the resistance $\rho \sim B^{\alpha}$ in this situation where $1<\alpha<2$ for the generic direction in $\Pi(\mathbf{B})$.

On the Fig.14 (Fig.11 in [7]) we can see series of black domains where $B^{2}$ - dependence has been observed.

It is interesting to point out that in the centers of the black domains we find dots where the resistance has "very deep minima" and correspond to the Case 1, according to the results of [7]. This situation is precisely analogous to the case of the biggest stability zones for the analytic dispersion relation (5) and we have the "singular nets" in the planes orthogonal to these "Special rational directions".

The resistance within the black domains should be the $B^{2}$ - type, like in the Case 2. However, this dependence was found experimentally like $B^{\alpha}$ for $\alpha \leq 2$ ("slightly less", as written in [7]). Probably, magnetic field $B \sim 1 T$ was not enough for our asymptotic behavior. We think that these experi- 


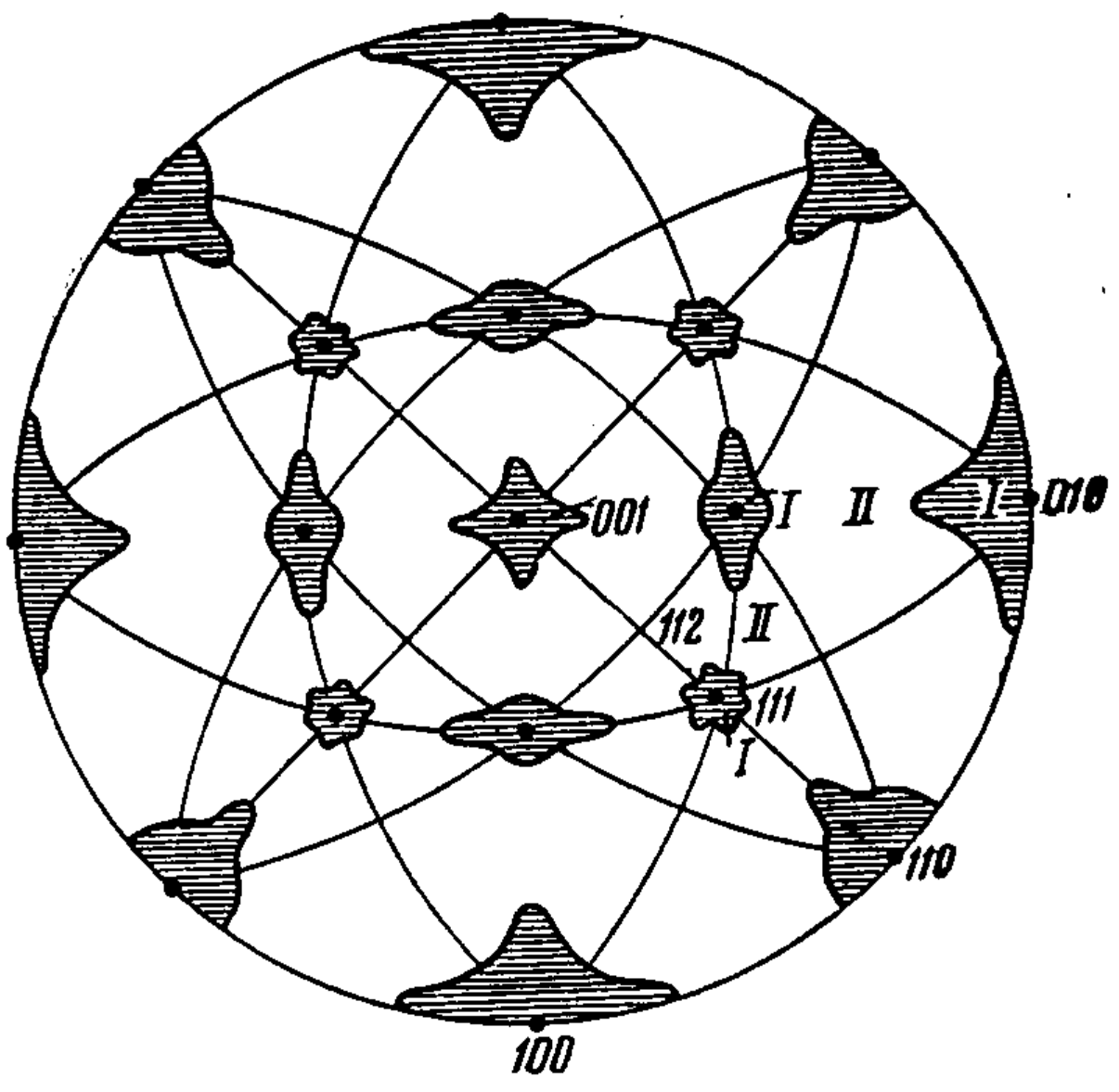

Figure 14: Experimental data obtained by Yu.P.Gaidukov for Au. Black domains correspond to Case 2. 
ments should be repeated with $B \sim 10 T$ to get the precise $B^{2}$-dependence. In this case we can definitely state that these black domains are really the "Stability Zones" whose topological types correspond to the integral planes orthogonal to unit vectors directed into the central points. So we predict here the following set of the "Topological Quantum numbers":

$$
\begin{gathered}
\left(M_{1}, M_{2}, M_{3}\right)=( \pm 1,0,0),(0, \pm 1,0),(0,0, \pm 1),( \pm 1, \pm 1,0), \\
( \pm 1,0, \pm 1),(0, \pm 1, \pm 1),( \pm 1, \pm 1, \pm 1)
\end{gathered}
$$

Everywhere in the white area we have the Case 1 and the finite resistance as $B \rightarrow \infty$ in $\Pi(\mathbf{B})$.

The interesting behavior was found also along the black lines on the Fig 14.

Namely, in many points of these lines the resistance has very deep maxima where the asymptotic behavior like $B^{\alpha}$ has been observed for the different values of $\alpha$ : $1<\alpha<1$.8. In our opinion, these experimental results should be improved for $B \geq 10 T$ instead of $B \sim 2 T$ like in [7].

Conjecture. The non-generic chaotic orbits exist here.

Unfortunately the conductivity along the magnetic field $\mathbf{B}$ was not measured in [7] so we do not have any information if any local minima of longitudinal conductivity were observed for these special directions.

\section{References}

[1] I.M.Lifshitz, M.Ya.Azbel, M.I.Kaganov. Sov. Phys. JETP 4, 41 (1957).

[2] I.M.Lifshitz, V.G.Peschansky. Sov. Phys. JETP 8, 875 (1959).

[3] I.M.Lifshitz, V.G.Peschansky. Sov. Phys. JETP 11, 137 (1960).

[4] N.E.Alexeevsky, Yu.P.Gaidukov. Sov. Phys. JETP 8, 383 (1959).

[5] N.E.Alexeevsky, Yu.P.Gaidukov. Sov. Phys. JETP 9, 311 (1959).

[6] N.E.Alexeevsky, Yu.P.Gaidukov. Sov. Phys. JETP 10, 481 (1960).

[7] Yu.P.Gaidukov. Sov. Phys. JETP 10, 913 (1960).

[8] I.M.Lifshitz, M.I.Kaganov. Sov. Phys. Usp. 2, 831 (1960). 
[9] I.M.Lifshitz, M.I.Kaganov. Sov. Phys. Usp. 5, 411 (1962).

[10] I.M.Lifshitz, M.Ya.Azbel, M.I.Kaganov. Electron Theory of Metals. Moscow, Nauka, 1971. Translated: New York: Consultants Bureau, 1973.

[11] A.A.Abrikosov. Fundamentals of the Theory of Metals. "Nauka", Moscow (1987). Translated: Amsterdam: North-Holland, 1998.

[12] S.P.Novikov. Russian Math. Surveys 37 (1982).

[13] S.P.Novikov. Trudy MIAN. 1984. 166, 201-209. (Proc. Steklov Inst. Math. 1 (1986)).

[14] S.P.Novikov. "Quasiperiodic structures in topology". Proc. Conference "Topological Methods in Mathematics", dedicated to the 60th birthday of J.Milnor, June 15-22, S.U.N.Y. Stony Brook, 1991. Publish of Perish, Houston, TX, pp. 223-233 (1993).

[15] S.P.Novikov. Proc. Conf. of Geometry, December 15-26, 1993, Tel Aviv University (1995).

[16] A.V.Zorich. Uspekhi Mat.Nauk 39:5, 235-236 (1984). (Russian Math. Surveys 39 (1984).)

[17] I.A.Dynnikov. Uspekhi Mat. Nauk 47:3 (285), 161-162 (1992). (Russian Math. Surveys 47 (1992).)

[18] I.A.Dynnikov. Uspekhi Mat. Nauk 48:2 (290), 179-180 (1993). (Russian Math. Surveys 48 (1993).)

[19] I.A.Dynnikov. "A proof of Novikov's conjecture on semiclassical motion of electron." Mat. Zametki 53:5, 57 (1993). (Math. Notes 53:5, 495-501 (1993).)

[20] S.P.Novikov, A.Ya.Maltsev. ZhETP Lett. 63, 855 (1996).

[21] I.A.Dynnikov. "Surfaces in 3-Torus: Geometry of plane sections." Proc.of ECM2, BuDA, 1996. 
[22] I.A.Dynnikov. Dissertation. Moscow State University of M.V.Lomonosov, Scientific Supervisor - S.P.Novikov, Moscow (1996).

[23] I.A.Dynnikov. "Semiclassical motion of the electron. A proof of the Novikov conjecture in general position and counterexamples." American Mathematical Society Translations, Series 2, Vol. 179, Advances in the Mathematical Sciences. Solitons, Geometry, and Topology: On the Crossroad. Editors: V.M.Buchstaber, S.P.Novikov. (1997)

[24] I.A.Dynnikov, A.Ya.Maltsev. ZhETP 85, 205 (1997).

[25] A.Ya. Maltsev. ZhETP 85, 934 (1997).

[26] S.P.Novikov, A.Ya.Maltsev. Physics-Uspekhi 41(3), 231-239 (1998).

[27] A.V.Zorich. Proc. "Geometric Study of Foliations" (Tokyo, November 1993)/ ed. T.Mizutani et al. Singapore: World Scientific, 479-498 (1994).

[28] I.A.Dynnikov. Uspekhi Mat.Nauk 54:1, 21-61 (1999). (Russian Math. Surveys 54 (1999).)

[29] S.P.Tsarev. Private communication. (1992-93).

[30] S.P.Novikov. Uspekhi Mat. Nauk 54:3 (329), 147-148 (1999) (Russian Math. Surveys 54:3, (1999)).

[31] R.D.Leo. PhD Theses. University of Maryland. Department of Math., College Park, MD 20742, USA.

[32] V.I.Arnold. Functional analysis and its appilcations bf 25:2 (1991).

[33] Ya.G.Sinai, K.M.Khanin. Functional analysis and its appilcations bf 26:3 (1992).

[34] A.B.Pippard. Phil. Trans. Roy. Soc., A250, 325 (1957).

[35] M.H.Cohen, L.M.Falicov. Phys. Rev. Lett. 7, 231 (1961). 